\title{
Quantitative Stability Analysis of Stochastic Quasi-Variational Inequality Problems and Applications
}

\author{
Jie Zhang ${ }^{1}$ Huifu $\mathrm{Xu}^{2}$ and Li-wei Zhang ${ }^{3}$
}

December 6, 2016

\begin{abstract}
We consider a parametric stochastic quasi-variational inequality problem (SQVIP for short) where the underlying normal cone is defined over the solution set of a parametric stochastic cone system. We investigate the impact of variation of the probability measure and the parameter on the solution of the SQVIP. By reformulating the SQVIP as a natural equation and treating the orthogonal projection over the solution set of the parametric stochastic cone system as an optimization problem, we effectively convert stability of the SQVIP into that of a one stage stochastic program with stochastic cone constraints. Under some moderate conditions, we derive Hölder outer semicontinuity and continuity of the solution set against the variation of the probability measure and the parameter. The stability results are applied to a mathematical program with stochastic semidefinite constraints and a mathematical program with SQVIP constraints.
\end{abstract}

Key words. Stochastic quasi-variational inequality, quantitative stability analysis, mathematical program with stochastic semidefinite constraints, mathematical program with SQVIP constraints

AMS Subject Classifications. 90C15, 90C30, 90C33.

\section{Introduction}

Let $F: \mathbb{R}^{n} \times \mathbb{R}^{m} \times \mathbb{R}^{k} \rightarrow \mathbb{R}^{m}$ be a continuous function and $\xi: \Omega \rightarrow \Xi$ be a random vector with $k$ components defined on a probability space $(\Omega, \mathcal{F}, P)$, let $\mathcal{Y} \subset \mathbb{R}^{m}$ be a closed convex set. We consider the following parametric stochastic quasi-variational inequality problem (SQVIP): for a fixed parameter $x_{0} \in \mathbb{R}^{n}$, find $y \in \mathcal{Y}$ such that

$$
0 \in \mathbb{E}_{P}\left[F\left(x_{0}, y, \xi(\omega)\right)\right]+\mathcal{N}_{\Gamma_{P}\left(x_{0}, y\right)}(y),
$$

where $\mathbb{E}_{P}[\cdot]$ denotes the mathematical expectation with respect to the probability measure $P$ and $\Gamma_{P}: \mathbb{R}^{n} \times \mathbb{R}^{m} \rightarrow 2^{\mathbb{R}^{m}}$ is a closed set-valued mapping associated with $P$ (to be clarified shortly), and $\mathcal{N}_{\Gamma_{P}\left(x_{0}, y\right)}(y)$ is the normal cone of $\Gamma_{P}\left(x_{0}, y\right)$ at $y$ in the sense of convex analysis. Throughout the paper, we assume that $\mathbb{E}_{P}[F(x, y, \xi)]$ is well defined for every $x \in \mathbb{R}^{n}$ and $y \in \mathbb{R}^{m}$. Note that if we consider $(\Xi, \mathscr{B})$ as a measurable space equipped with Borel sigma algebra $\mathscr{B}$, then $P$ may be viewed as a probability measure defined on $(\Xi, \mathscr{B})$ induced by the random variate $\xi$. Consequently, we may drop $\omega$ in the mathematical expectation.

\footnotetext{
${ }^{1}$ School of Mathematics, Liaoning Normal University, Dalian 116029, China. (e-mail:zhangjie04212001@163.com). The research of this author is supported by the NSFC under project No.11671183 and No.11201210, CPSF under project No.2014M560200 and Program for Liaoning Excellent Talents in University under project No. LJQ2015059.

${ }^{2}$ School of Mathematical Science, University of Southampton, SO17, 1BJ, Southampton, UK. (email:H.Xu@soton.ac.uk), Haitian Scholar, Dalian University of Technology.

${ }^{3}$ Institute of ORCT, School of Mathematical Sciences, Dalian University of Technology, Dalian 116024, China. (email:lwzhang@dlut.edu.cn). The research of this author was supported by the NSFC under project No. 91330206 and No. 11571059.
} 
In practice, $\Gamma_{P}$ may take a closed form in the sense that it has an explicit structure such as Aumann's integral of a random set-valued mapping [2]. It could also be implicitly defined as the set of solutions to a stochastic system of equalities and inequalities. Here we consider the latter case but in a slightly more general fashion with $\Gamma_{P}(x, y)$ being the set of solutions to the following stochastic cone system:

$$
\Gamma_{P}(x, y)=\left\{z \in \mathcal{Z}: \mathbb{E}_{P}[G(x, y, z, \xi)] \in \mathcal{K}\right\},
$$

where $\mathcal{Z} \subset \mathcal{Y}$ is a closed convex set and $\mathcal{K}$ is a closed convex cone in a vector or a matrix space denoted by $W$, and $G$ is a continuous mapping from $\mathbb{R}^{n} \times \mathbb{R}^{m} \times \mathbb{R}^{m} \times \Xi$ to $W$. To simplify the discussion, we assume $\mathcal{Z}=\mathcal{Y}$ and $\Gamma_{P}(x, y)$ is a convex set for each fixed $(x, y)$. A sufficient condition is that for each $(x, y, \xi) \in \mathbb{R}^{n} \times \mathbb{R}^{m} \times \Xi, G(x, y, \cdot, \xi): \mathbb{R}^{m} \rightarrow W$ is a $(-\mathcal{K})$-convex mapping (see [27]) on $\mathcal{Z}$, that is, for every pair of points $z_{1}, z_{2} \in \mathcal{Z}$ and any positive scalar $t \in(0,1)$, we have

$$
t \mathbb{E}_{P}\left[G\left(x, y, z_{1}, \xi\right)\right]+(1-t) \mathbb{E}_{P}\left[G\left(x, y, z_{2}, \xi\right)\right]-\mathcal{K} \subseteq \mathbb{E}_{P}\left[G\left(x, y, t z_{1}+(1-t) z_{2}, \xi\right)\right]-\mathcal{K} .
$$

The SQVIP model (1.1) is an extension of deterministic QVIPs ([6, 22, 21]) to address underlying uncertainties in various equilibrium problems arising from economics, transportation, structural balance and electrical power flow networks [13].

In a particular case when $\Gamma_{P}(x, y)$ is independent of $\mathbb{E}_{P}[\cdot], x$ and $y,(1.1)$ reduces to stochastic VIP (SVIP for short) which has been intensively studied over the past few years from numerical schemes such as stochastic approximation method and Monte Carlo method to the fundamental theory and applications; see for instance $[13,39,40,20,25,41,42]$ and the references therein.

At this point, we should remind the readers that the SVIP models we are discussing here are expected value based and they should be distinguished from another class of SVIP models proposed by Chen and Fukushima [7] where a deterministic solution is sought through minimization of the expected residual of scenario based SVIP, see [8] for the recent development of the latter. A new SVIP model which captures the features of the two SVIP models is recently proposed by Rockafellar and Wets [32].

When the cone system (1.2) satisfies some appropriate constraint qualifications and $G$ is continuously differentiable in $z$, it is possible to transform SQVIP (1.1) into a standard SVIP, see discussions by Pang and Fukushima [22] for deterministic cases. To avoid overlap with the existing research on SVIP, here we concentrate our discussion on the case when it is difficult to transfer the SQVIP to a SVIP either because $G$ is not continuously differentiable or it is difficult to represent $\mathcal{N}_{\Gamma_{P}\left(x_{0}, y\right)}(y)$ in terms of the normal cone of $\mathcal{K}$. Specifically we address the complications arising from the dependence of $\Gamma_{P}(x, y)$ on the underlying probability distribution, parameter $x$ and variable $y$ when we come down to approximation schemes in relation to $P$ and the underlying theory for the SQVIP. Instead of looking into a particular approximation scheme for the SQVIP, here we consider variation of the probability measure $P$ ( under some metric to be defined in Section 2) and its impact on the set of the solutions. This allows our results to cover a wide range of approximation schemes related to $P$ for the SQVIP.

This kind of research is known as stability analysis or perturbation analysis in the literature of optimization and variational analysis. While it is impossible for us to present a thorough literature review on this topic here, we note that a number of important results for stability analysis of deterministic parametric optimization problems are established by Robinson in his earlier works [29, 30, 31] and Klatte [16] etc., see monographs $[17,5]$ for a comprehensive review. In a more recent development, interesting progresses have been made on cone constrained optimization and quasi-variational 
inequalities, for instances, Arutyunov and Izmailov [1] derived some sensitivity results for mathematical programs with cone constraints such as first and second-order optimality conditions; Mordukhovich and Outrata [21] obtained efficient conditions for robust Lipschitzian stability of quasi-variational inequalities by exploiting coderivative of the solution maps; Izmailov, Kurennoy, and Solodov [12] used graphical derivative criterion to characterize the upper Lipschitz stability for Lipschitz continuous KKT systems; Ding, Sun and Zhang [9] characterized the isolated calmness of KKT solution mapping for a class of conic programming problems.

In stochastic programming, one of the main challenges is to tackle the mathematical expectation because in practice it is often difficult to obtain a closed form of the expected value of a random function. Various approximation schemes such as sample average approximation (SAA) or numerical integration have been proposed to address the challenge, we refer interested readers to the book chapters by Pflug and Shapiro in [34] for overviews of convergence analysis. An alternative way to analyze the approximation schemes and their impact on the underlying stochastic programming problems is to look into variation of the probability measure and investigate its impact from stability point of view. The approach is widely known as stability analysis in the literature of stochastic programming, see an excellent overview by Römisch [33].

Stability analysis of stochastic variational inequalities can be traced down to King and Rockafellar's early works $[14,15]$ which are partly motivated by the asymptotic analysis of statistical estimators in stochastic programming. More recently, Liu, Römish and Xu [18] presented some quantitative stability of the solution of the following stochastic generalized equations (SGE):

$$
0 \in \mathbb{E}_{P}[H(y, \xi)]+\mathcal{G}(y)
$$

where $H(\cdot, \cdot)$ and $\mathcal{G}(\cdot)$ are set-valued mappings. It is easy to see that (1.3) covers a wide range of interesting problems including SVIP and first order optimality conditions of one stage and two stage stochastic programming problems.

Unfortunately the SGE model does not cover (1.1) and this is indeed one of the main reasons for us to write up this paper because SQVIP is of independent interest. To see the relationship between the two formulations, let $\xi^{\prime}=(x, \xi), \delta_{x_{0}}(\cdot)$ denote the Dirac probability measure on $\mathbb{R}^{n}$ and $\pi_{0}:=\delta_{x_{0}} \times P$. Then we may write (1.1) as

$$
0 \in \mathbb{E}_{\pi_{0}}\left[F\left(y, \xi^{\prime}\right)\right]+\mathcal{N}_{\Gamma_{\pi_{0}}(y)}(y) .
$$

The fundamental difference between (1.3) and (1.1) is that the normal cone in the latter formulation depends on the probability distribution. For similar reasons, the results established in Liu, Xu and Lin [19] cannot be applied to stability analysis of (1.1). Thus, to carry out quantitative stability of SQVIP (1.1), we have to deal with new challenges arising from dependence of $\mathcal{N}_{\Gamma_{P}(x, y)}(y)$ on $P$ and $x$, and we propose to do so via the natural maps in the literature of VIP (see [11]), i.e., we reformulate the SQVIP (1.1) as

$$
\mathbf{F}_{P}^{\text {nat }}\left(x_{0}, y\right):=\Pi_{\Gamma_{P}\left(x_{0}, y\right)}\left(y-\mathbb{E}_{P}\left[F\left(x_{0}, y, \xi\right)\right]\right)-y=0,
$$

where $\Pi_{C}(w)$ denotes the orthogonal projection of $w$ on $C$ for a nonempty closed convex set $C$, $\mathbf{F}_{P}^{\text {nat }}\left(x_{0}, y\right)$ is the natural map associated with (1.1).

Let $Q$ denote a perturbation of the probability measure $P$. We consider a perturbation of the stochastic quasi variational inequality problem (1.1) with $P$ being replaced by $Q$ : given a parameter $x \in \mathbb{R}^{n}$, find a decision vector $y \in \mathcal{Y}$ such that

$$
0 \in \mathbb{E}_{Q}[F(x, y, \xi)]+\mathcal{N}_{\Gamma_{Q}(x, y)}(y),
$$


where $\Gamma_{Q}: \mathbb{R}^{n} \times \mathbb{R}^{m} \rightarrow 2^{\mathbb{R}^{m}}$ is defined by

$$
\Gamma_{Q}(x, y)=\left\{z \in \mathcal{Y}: \mathbb{E}_{Q}[G(x, y, z, \xi)] \in \mathcal{K}\right\} .
$$

Using the natural map, we can reformulate (1.6) as

$$
\mathbf{F}_{Q}^{\text {nat }}(x, y):=\Pi_{\Gamma_{Q}(x, y)}\left(y-\mathbb{E}_{Q}[F(x, y, \xi)]\right)-y=0 .
$$

Let $S_{P}\left(x_{0}\right)$ and $S_{Q}(x)$ denote the sets of solutions to problems (1.1) and (1.6) respectively. We investigate the relationship between $S_{Q}(x)$ and $S_{P}\left(x_{0}\right)$ as $Q$ approximates $P$ under some appropriate metric and parameter $x$ converges to $x_{0}$.

One of the advantages of the reformulation is that the projection map $\Pi_{\Gamma_{Q}(x, y)}\left(y-\mathbb{E}_{Q}[F(x, y, \xi)]\right)$ is single and finite valued and hence it may be relatively easier to handle compared to the normal cone $\mathcal{N}_{\Gamma_{Q}(x, y)}(y)$ (which is set-valued and unbounded). A key step for the stability analysis is to quantify the gap between $\Pi_{\Gamma_{Q}(x, y)}\left(y-\mathbb{E}_{Q}[F(x, y, \xi)]\right)$ and $\Pi_{\Gamma_{P}\left(x_{0}, y\right)}\left(y-\mathbb{E}_{P}\left[F\left(x_{0}, y, \xi\right)\right]\right)$. Since the projection can be reformulated as the solution to a parametric stochastic minimization problem with cone constraints (1.2), we quantify the difference by investigating stability of the optimal solutions of the latter. As far as we are concerned, the main contributions of this paper can be summarized as follows.

- We carry out quantitative stability analysis for a deterministic parametric minimization problem with cone constraints. Under the Slater constraint qualification, we derive Hölder continuity for the feasible solution set mapping (Lemma 2.2 ) and the optimal solution set mapping against variation of the parameter over Banach space (Theorem 2.1). In comparison with the existing stability results for parametric programming (see e.g [5, Chapter 4], [36], [35] and [1]), our results are established without any assumption on continuous differentiability of the underlying functions or reducibility of $\mathcal{K}$.

- We apply the stability result to quantify the discrepancy between $\Pi_{\Gamma_{P}\left(x_{0}, y\right)}\left(y-\mathbb{E}_{P}\left[F\left(x_{0}, y, \xi\right)\right]\right)$ and $\Pi_{\Gamma_{Q}(x, y)}\left(y-\mathbb{E}_{Q}[F(x, y, \xi)]\right)$ and its propagation to the solutions of (1.5) and (1.8) under the Slater constraint qualification of parametric cone system (1.2). Moreover, under some metric regularity conditions and Lipschitz homeomorphism of the natural map, we obtain a linear error bound for the solution set mapping with respect to variation of the probability measures under pseudometric (Theorem 4.1). Prior to the stability analysis, we also investigated sufficient conditions for existence of solutions to perturbed SQVIP (1.6) through (1.5)(Theorem 3.1).

- We apply the stability results to a mathematical program with stochastic semidefinite constraints and a mathematical program with SQVIP constraints. The former provides a new stochastic model which has potential applications in portfolio optimization and the latter extends existing research on stochastic mathematical programs with equilibrium constraints, see details in Section 5 .

The rest of the paper is organized as follows. Section 2 introduces some well known concepts and results in set-valued analysis and stochastic programming needed in this paper. It also presents some new quantitative stability results for a deterministic parametric minimization problem with cone constraints to be used for the stability analysis of SQVIP. Section 3 addresses existence of solutions to the perturbed SQVIP (1.6) followed by the main stability analysis in Section 4. Section 5 applies the established stability results in Section 4 to two new classes of stochastic optimization models. 


\section{Preliminaries}

In this section, we make preparations for the main quantitative stability analysis of SQVIP outlined in the introduction. Specifically, we introduce appropriate metrics which can be effectively used to quantify the variation/perturbation of the underlying probability measure as well as the tool for quantifying the gap between $\Pi_{\Gamma_{Q}(x, y)}\left(y-\mathbb{E}_{Q}[F(x, y, \xi)]\right)$ and $\Pi_{\Gamma_{P}\left(x_{0}, y\right)}\left(y-\mathbb{E}_{P}\left[F\left(x_{0}, y, \xi\right)\right]\right)$. For the first purpose, we consider the pseudo metric which is widely used in stability analysis of stochastic programming and for the second purpose, we derive a generic quantitative stability result for a parametric nonlinear program which can be easily used to quantify the specified gap. We will also recall some basic concepts and elementary results in set-valued and variational analysis including metric regularity, epiconvergence and Robinson-Ursescu stability theorem.

\section{$2.1 \quad$ Notation}

Throughout this paper, we use the following notation. By convention, $\mathbb{R}^{n}$ stands for $n$-dimensional Euclidean space. For a real-valued function $\varphi: \mathbb{R}^{n} \rightarrow \mathbb{R} \cup\{ \pm \infty\}$, epi $\varphi$ denotes its epigraph, that is, the set $\left\{(x, \alpha) \in \mathbb{R}^{n} \times \mathbb{R}: \varphi(x) \leq \alpha\right\}$. For a set-valued mapping $\Phi: X \rightarrow 2^{Y}$, we write $\operatorname{gph} \Phi:=\{(x, y) \in X \times Y: y \in \Phi(x), x \in X\}$ for its graph and range $\Phi:=\{y \in Y: Y \in \Phi(x), x \in X\}$ for its range. By convention int $S, \operatorname{cl} S$ bd $S$ the interior, closure, and boundary of $S$. We use $B_{n}(x, \delta)$ to denote a closed ball with center $x \in \mathbb{R}^{n}$ and radius $\delta$ and $\mathcal{B}_{n}$ specifically for the closed unit ball centered at 0 in $\mathbb{R}^{n}$. We let $d(x, S):=\inf _{x^{\prime} \in S}\left\|x-x^{\prime}\right\|$ denote the distance from point $x \in \mathbb{R}^{n}$ to a set $S \subset \mathbb{R}^{n}$. For two sets $S_{1}, S_{2} \subset \mathbb{R}^{n}$,

$$
\mathbb{D}\left(S_{1}, S_{2}\right):=\inf \left\{t \geq 0: S_{1} \subset S_{2}+t \mathcal{B}_{n}\right\}
$$

signifies the deviation of $S_{1}$ from $S_{2}$ and $\mathbb{H}\left(S_{1}, S_{2}\right):=\max \left(\mathbb{D}\left(S_{1}, S_{2}\right), \mathbb{D}\left(S_{2}, S_{1}\right)\right)$ the Hausdorff distance between the two sets. Finally, for a sequence of subsets $\left\{S_{k}\right\}$ in a metric space, we follow the standard notation (see [3, Definition 1.1.1]) by using $\lim \sup _{k \rightarrow \infty} S_{k}$ to denote its upper limit, that is,

$$
\limsup _{k \rightarrow \infty} S_{k}=\left\{x: \liminf _{k \rightarrow \infty} d\left(x, S_{k}\right)=0\right\}
$$

and $\lim \inf _{k \rightarrow \infty} S_{k}$ to denote its lower limit, that is,

$$
\liminf _{k \rightarrow \infty} S_{k}=\left\{x: \lim _{k \rightarrow \infty} d\left(x, S_{k}\right)=0\right\}
$$

It is well known that $\liminf _{k \rightarrow \infty} S_{k} \subseteq \lim \sup _{k \rightarrow \infty} S_{k}$, see [3].

\subsection{Some basic concepts and results in set-valued and variational analysis}

Let $\Psi: \mathbb{R}^{n} \rightarrow 2^{\mathbb{R}^{m}}$ be a set-valued mapping. Recall that $\Psi$ is said to be closed at $\bar{x}$ if $x_{k} \in X, x_{k} \rightarrow \bar{x}$, $y_{k} \in \Psi\left(x_{k}\right)$ and $y_{k} \rightarrow \bar{y}$ implies $\bar{y} \in \Psi(\bar{x})$. $\Psi$ is said to be upper semi-continuous (usc for short) at $\bar{x} \in \mathbb{R}^{n}$ if and only if for any neighborhood $\mathcal{U}$ of $\Psi(\bar{x})$, there exists a positive number $\delta>0$ such that for any $x^{\prime} \in B_{n}(x, \delta) \cap \mathbb{R}^{n}, \Psi\left(x^{\prime}\right) \subset \mathcal{U}$. When $\Psi$ is closed at $\bar{x}$ and $\Psi(\bar{x})$ is compact, $\Psi$ is upper semicontinuous at $\bar{x}$ if and only if for every $\epsilon>0$, there exists a constant $\delta>0$ such that

$$
\Psi\left(\bar{x}+\delta \mathcal{B}_{n}\right) \subset \Psi(\bar{x})+\epsilon \mathcal{B}_{m} .
$$


$\Psi$ is said to be lower semi-continuous (lsc for short) at $\bar{x} \in \mathbb{R}^{n}$ if and only if for any $\bar{y} \in \Psi(\bar{x})$ and any sequence $\left\{x_{k}\right\} \subset \mathbb{R}^{n}$ converging to $\bar{x}$, there exists a sequence $\left\{y_{k}\right\}$, where $y_{k} \in \Psi\left(x_{k}\right)$, converging to $\bar{y}$. The lower semicontinuity holds if and only if for any open set $\mathcal{U}$ with $\mathcal{U} \cap \Psi(\bar{x}) \neq \emptyset$, the set $\{x \in X: \mathcal{U} \cap \Psi(x) \neq \emptyset\}$ is a neighborhood of $\bar{x}$. $\Psi$ is said to be continuous at $\bar{x}$ if it is both usc and lsc at the point; see [3] for details.

Note that in the literature of set-valued analysis, there are other notions describing semi-continuity of a set-valued mapping. For instances, Rockafellar and Wets [26, Definiton 5.4] introduce the concept of inner semicontinuity and the outer semicontinuity and assert that when a set-valued mapping is locally bounded, usc coincides with outer semicontinuity and lsc agrees with inner semicontinuity, see commentary of [26, Chapter 5].

We also need the following definition.

Definition 2.1 [26, Definition 7.39] For $f: \mathbb{R}^{n} \times \mathbb{R}^{m} \rightarrow \mathbb{R} \cup\{ \pm \infty\}$, the function-valued mapping $u \mapsto f(\cdot, u)$ is epi-continuous at $\bar{u}$, written

$$
f(\cdot, \bar{u})=e-\lim _{u \rightarrow \bar{u}} f(\cdot, u)
$$

if the set-valued mapping $u \mapsto$ epi $f(\cdot, u)$ is continuous at $\bar{u}$.

The characterization of the epi-continuity can be described by the following result.

Proposition 2.1 [26, Exercise 7.40] For $f: \mathbb{R}^{n} \times \mathbb{R}^{m} \rightarrow \mathbb{R} \cup\{ \pm \infty\}$, the function-valued mapping $u \mapsto f(\cdot, u)$ is epi-continuous at $\bar{u}$ if and only if for every sequence $u^{\nu} \rightarrow \bar{u}$ and point $\bar{x} \in \mathbb{R}^{n}$, the following two conditions hold:

(a) $\liminf _{\nu \rightarrow \infty} f\left(x^{\nu}, u^{\nu}\right) \geq f(\bar{x}, \bar{u})$ for every sequence $x^{\nu} \rightarrow \bar{x}$,

(b) $\quad \lim \sup _{\nu \rightarrow \infty} f\left(x^{\nu}, u^{\nu}\right) \leq f(\bar{x}, \bar{u})$ for some sequence $x^{\nu} \rightarrow \bar{x}$.

One of the main tasks of set-valued and variational analysis is to detect stability of nonlinear systems when the underlying decision variables and/or parameters perturb. In the latter case, the perturbation is often associated with problem data defining the system. For this purpose, we need the notion of metric regularity which is well established in the literature (see e.g. [5]).

Definition 2.2 Let $\Psi: \mathbb{R}^{n} \rightarrow 2^{\mathbb{R}^{m}}$ be a set-valued mapping. $\Psi$ is said to be metrically regular at a point $\left(x_{0}, y_{0}\right) \in \operatorname{gph} \Psi$, with modulus $C$, if for all $(x, y)$ in a neighborhood of $\left(x_{0}, y_{0}\right)$,

$$
d\left(x, \Psi^{-1}(y)\right) \leq C d(y, \Psi(x)) .
$$

A powerful result arising from metric regularity is Robinson-Ursescu theorem which effectively relates the well known Slater type constraint qualification to metric regularity of a set-valued mapping.

Lemma 2.1 (Robinson-Ursescu stability theorem [5]) Let $\Psi: \mathbb{R}^{n} \rightarrow 2^{\mathbb{R}^{m}}$ be a closed convex setvalued mapping. Then $\Psi$ is metrically regular at $\left(x_{0}, y_{0}\right) \in \mathrm{gph} \Psi$ if and only if the regularity condition

$$
y_{0} \in \text { int range } \Psi \text {. }
$$


More precisely, there exist positive constants $\nu$ and $\eta$ such that (2.2) implies (2.1) with $C=4 \nu / \eta$ when

$$
\left\|x-x_{0}\right\| \leq \frac{1}{2} \nu ; \quad\left\|y-y_{0}\right\| \leq \frac{1}{8} \eta
$$

When $\Psi$ is single-valued, we need the following concept of locally Lipschitz Homeomorphism.

Definition 2.3 A continuous function $\Psi: \mathcal{O} \subseteq \mathbb{R}^{n} \rightarrow \mathbb{R}^{n}$ is said to be locally Lipschitz invertible near $x \in \mathcal{O}$ if there exists an open neighborhood $\mathcal{N} \subseteq \mathcal{O}$ of $x$ such that the restricted map $\left.\Psi\right|_{\mathcal{N}}: \mathcal{N} \rightarrow \Psi(\mathcal{N})$ is bijective and its inverse is Lipschitz continuous. $F$ is said to be a locally Lipschitz homeomorphism near $x$ if $\Psi$ is locally Lipschitz invertible near $x$ and $\Psi$ itself is locally Lipschitz continuous near $x$.

\subsection{Quantitative stability analysis for deterministic parametric programs}

Let $U$ be a metric space equipped with norm $\|\cdot\|_{U}$, and let $\phi$ and $\psi$ be continuous functions from $\mathbb{R}^{m} \times U$ to $\mathbb{R}$ and $W$ respectively. We consider the following parametric minimization problem:

$$
\begin{array}{cl}
\min _{z \in \mathbb{R}^{m}} & \phi(z, u) \\
\left(\mathcal{P}_{u}\right) \quad \text { s.t. } & \psi(z, u) \in \mathcal{K}, \\
& z \in \mathcal{Z},
\end{array}
$$

where $u \in U$ is a fixed parameter, $\mathcal{Z} \subset Y$ is a compact set and $\mathcal{K}$ is a closed convex cone in the space of $W$ equipped with norm $\|\cdot\|_{W}$. Unless specified otherwise, we do not assume differentiability of $\psi$ or $\phi$.

Let $\Psi(u), \mathcal{Z}(u)$ and $\vartheta(u)$ denote respectively the set of feasible solutions, the set of the optimal solutions and the optimal value of $\mathcal{P}_{u}$. Assume $\Psi(u) \neq \emptyset$ for each $u$ concerned. Since $\mathcal{Z}$ is a compact set, and $\phi(\cdot, u)$ and $\psi(\cdot, u)$ are continuous functions, then $\mathcal{Z}(u)$ is a nonempty compact set and $\vartheta(u)$ is finite valued.

We investigate stability of the parametric program by considering a perturbation of parameter $u$ in a neighborhood of $\bar{u}$ and quantifying its impact on the optimal value and the optimal solutions. At this point, we note that quantitative stability analysis of parametric programs is well documented, see monograph by Bonnans and Shapiro [5]. Here we concentrate on the case when the underlying functions are not necessarily differentiable.

To this end, we first study stability of the feasible set $\Psi(u)$. If we regard $\Psi(\cdot)$ as a set-valued mapping, then the research is in essence down to characterizing continuity of the set-valued mapping at $\bar{u}$ and quantifying the difference between $\Psi(u)$ and $\Psi(\bar{u})$ when $u$ is sufficiently close to $\bar{u}$. Without loss of generality, we assume that $\bar{u}$ and its perturbation $u$ are restricted to a compact set $\mathcal{U} \subset U$.

Lemma 2.2 Assume: (a) $\psi(\cdot, u): \mathcal{Z} \rightarrow W$ is a continuous $(-\mathcal{K})$-convex function; (b) $\psi(z, u)$ is continuous in $u$ at $\bar{u}$ uniformly w.r.t. $z$; (c) problem (2.3) satisfies the following Slater constraint qualification (SCQ for short) for $u=\bar{u}$ :

$$
0 \in \operatorname{int}\{\psi(\mathcal{Z}, \bar{u})-\mathcal{K}\},
$$

where $\psi(\mathcal{Z}, \bar{u}):=\{\psi(z, \bar{u}): z \in \mathcal{Z}\}$. Then 
(i) $\Psi(\cdot)$ is continuous at $\bar{u}$;

(ii) if, in addition, (d) $\psi(z, u)$ is Hölder continuous in $u$ at $\bar{u}$ uniformly w.r.t. $z$, i.e., there exist some constants $\sigma>0, \varepsilon>0$ and $\nu \in(0,1)$ such that

$$
\|\psi(z, u)-\psi(z, \bar{u})\|_{W} \leq \sigma\|u-\bar{u}\|_{U}^{\nu}
$$

for all $u \in B(\bar{u}, \varepsilon)$, then there exists a positive constant $C$ such that

$$
\mathbb{H}(\Psi(u), \Psi(\bar{u})) \leq C\|u-\bar{u}\|_{U}^{\nu}
$$

for all $u \in B(\bar{u}, \varepsilon)$. Here and later on, $B(\bar{u}, \varepsilon):=\left\{u \in U:\|u-\bar{u}\|_{U} \leq \varepsilon\right\}$.

We defer the proof to the appendix as it is not the main focus of this paper.

It is important to note that both convexity of $\psi(\cdot, u)$ (w.r.t. $-\mathcal{K}$ ) and Slater constraint qualification are essential for the continuity of $\Psi(\cdot)$. Indeed, we can easily find a counter example that continuity may fail in the absence of either condition.

Example 2.1 Consider the following simple parametric cone system

$$
\psi(z, u) \in \mathcal{K},
$$

where

$$
\psi(z, u)= \begin{cases}|z|-1, & \text { for } z \leq 1 \\ u(z-1)^{2}, & \text { for } z \geq 1\end{cases}
$$

$u$ is a parameter, and $\mathcal{K}=(-\infty, 0]$. It is easy to see that $\psi(\cdot, u)$ is not convex. Let $u \in \mathcal{U}=[0,1]$ and $z$ is restricted to take values from $\mathcal{Z}:=[-2,2]$. Then

$$
\Psi(u):=\{z \in \mathcal{Y}: \psi(z, u) \leq 0\}= \begin{cases}{[-1,1],} & \text { for } u>0 \\ {[-1,2],} & \text { for } u=0 .\end{cases}
$$

It is easy to verify that $\Psi(\cdot)$ is usc at 0 but it is not lsc at the point. However, problem (2.6) satisfies the SCQ.

To see the necessity of SCQ, let us change $\psi(z, u)$ to the following:

$$
\psi(z, u)= \begin{cases}|z|-1, & \text { for } z<-1 \\ 0, & \text { for } z \in[-1,1) \\ u(z-1)^{2}, & \text { for } z \geq 1\end{cases}
$$

Consequently problem (2.6) fails to satisfy SCQ because its solution set does not have an interior. On the other hand, $\psi(\cdot, u)$ is convex. The solution set $\Psi(u)$, however, remains the same as defined in (2.7), which means $\Psi(\cdot)$ is not continuous at 0 .

Let $\mathcal{Z}(u)$ and $\mathcal{Z}(\bar{u})$ denote the respective sets of optimal solutions and $\vartheta(u)$ and $\vartheta(\bar{u})$ the corresponding optimal values. 
Lemma 2.3 Let conditions (a)-(c) in Lemma 2.2 hold. Then $\vartheta(\cdot)$ is continuous at $\bar{u}$ and $\mathcal{Z}(\cdot)$ is upper semicontinuous at $\bar{u}$.

Form the above lemma, there exist positive constants $\bar{\delta}_{z}, \bar{\delta}_{u}$ (independent of $z$ and $u$ ) such that

$$
\mathcal{Z}(u) \subseteq \mathcal{Z}(\bar{u})+\bar{\delta}_{z} \mathcal{B}_{m}, \forall u \in B\left(\bar{u}, \bar{\delta}_{u}\right)
$$

With Lemma 2.2 and Lemma 2.3, we are now ready to present quantitative stability results of parametric minimization problem (2.3).

Theorem 2.1 Let conditions (a)-(c) in Lemma 2.2 hold. Assume that the objective function $\phi$ satisfies the following: $(a) \phi(z, u)$ is Hölder continuous in $u$ at $\bar{u}$ uniformly w.r.t. $z$ in a neighborhood of $\mathcal{Z}(\bar{u})$, i.e., there exist some positive constants $\bar{\delta}_{z}, \bar{\delta}_{u}, \varrho$ and $\gamma \in(0,1)$ such that

$$
|\phi(z, u)-\phi(z, \bar{u})| \leq \varrho\|u-\bar{u}\|_{U}^{\gamma}
$$

for $u \in B\left(\bar{u}, \bar{\delta}_{u}\right)$ and $z \in \mathcal{Z}(\bar{u})+\bar{\delta}_{z} \mathcal{B}_{m} ;(b) \phi(z, u)$ is globally Lipschitz continuous in $z \in \mathcal{Z}(\bar{u})+\bar{\delta}_{z} \mathcal{B}_{m}$ with modulus $L$ uniformly for all $u \in B\left(\bar{u}, \bar{\delta}_{u}\right)$; (c) $\phi(\cdot, \bar{u})$ satisfies the second order growth condition at the optimal solution set $\mathcal{Z}(\bar{u})$, that is, there exists a positive constant $\alpha>0$ such that

$$
\phi(z, \bar{u})-\vartheta(\bar{u}) \geq \alpha d(z, \mathcal{Z}(\bar{u}))^{2}, \quad \forall z \in \Psi(\bar{u}) \cap\left(\mathcal{Z}(\bar{u})+\bar{\delta}_{z} \mathcal{B}_{m}\right) .
$$

Then

(i) there exists a positive constant $C$ such that

$$
\mathbb{D}(\mathcal{Z}(u), \mathcal{Z}(\bar{u})) \leq C\|u-\bar{u}\|_{U}^{\beta}
$$

for all $u \in B\left(\bar{u}, \bar{\delta}_{u}^{\prime}\right)$, where $\beta=\frac{1}{2} \min (\nu, \gamma), \bar{\delta}_{u}^{\prime}=\min \left\{\bar{\delta}_{u}, \varepsilon\right\}, \nu$ and $\varepsilon$ are defined as in Lemma 2.2.

(ii) If the second order growth condition holds uniformly for all $u \in B\left(\bar{u}, \bar{\delta}_{u}\right)$, then

$$
\mathbb{H}(\mathcal{Z}(u), \mathcal{Z}(\bar{u})) \leq C\|u-\bar{u}\|_{U}^{\beta}
$$

for all $u \in B\left(\bar{u}, \bar{\delta}_{u}^{\prime}\right)$.

We defer the proofs of Lemma 2.3 and Theorem 2.1 to the appendix as they are long and technical.

Theorem 2.1 gives rise to quantitative stability analysis for the parametric minimization problem (2.3) under standard conditions (see Klatte [16]) including uniform Hölder continuity of the constraint function $\psi$ w.r.t. parameter $u$, Slater constraint qualification and second order growth condition of the objective function. Specifically, it says that the set of the optimal solutions is locally upper Hölder continuous at $\bar{u}$ if $\psi(\cdot, u)$ is convex, and locally Hölder continuous at $\bar{u}$ if the second order growth condition is uniform w.r.t. parameter $u$. Compared to existing stability results (see e.g [5, Chapter 4], [36], [35] and [1]), the strength of this theorem is that it is established without any assumption on continuous differentiability of the underlying functions or reducibility of $\mathcal{K}$. The result has potential to be applied to semidefinite programming by setting $W$ to a matrix space and $\mathcal{K}$ to the cone of semidefinite matrices albeit it is not our primal goal in this paper. 
Let us now consider a special case of problem (2.3)

$$
\begin{array}{cl}
\min _{z \in \mathbb{R}^{m}} & \phi(z, u):=\frac{1}{2}\|z-g(u)\|^{2}, \\
\text { s.t. } & \psi(z, u) \in \mathcal{K} \\
& z \in \mathcal{Z}
\end{array}
$$

where $\mathcal{Z}$ is defined as in $(2.3)$ and $g(u)$ is a continuous function of $u$. It is easy to observe that the optimal solution to (2.11) is the orthogonal projection of $g(u)$ on the feasible set $\Psi(u)$, namely $\Pi_{\Psi(u)}(g(u))$.

Corollary 2.1 Consider parametric minimization problem (2.11). Assume that conditions (a)-(d) in Lemma 2.2 hold. Assume also that $g: \mathcal{U} \rightarrow \mathbb{R}^{m}$ is Hölder continuous at $\bar{u}$, that is, there exist positive constants @ and $\gamma \in(0,1)$ such that

$$
\|g(u)-g(\bar{u})\| \leq \varrho\|u-\bar{u}\|_{U}^{\gamma}, \forall u \in B\left(\bar{u}, \bar{\delta}_{u}\right),
$$

where $\bar{\delta}_{u}$ is a constant defined as in Theorem 2.1. Then there exists a positive constant $C^{\prime}$ such that

$$
\left\|\Pi_{\Psi(u)}(g(u))-\Pi_{\Psi(\bar{u})}(g(\bar{u}))\right\| \leq C^{\prime}\|u-\bar{u}\|_{U}^{\beta}, \forall u \in B\left(\bar{u}, \bar{\delta}_{u}^{\prime}\right),
$$

where $\beta=\frac{1}{2} \min (\nu, \gamma), \bar{\delta}_{u}^{\prime}=\min \left\{\bar{\delta}_{u}, \varepsilon\right\}$, and $\nu$ and $\varepsilon$ are defined as in Lemma 2.2.

Proof. It suffices to verify conditions of Theorem 2.1. Observe first that the set of optimal solutions $\mathcal{Z}(u)$ is a singleton because $\Psi(u)$ is a convex set and $\mathcal{Z}(u)=\left\{\Pi_{\Psi(u)}(g(u))\right\}$. Since the conditions on the constraints are identical to those in Theorem 2.1, we only need to verify the conditions for the objective function $\phi$. Here

$$
\phi(z, u)=\frac{1}{2}\left(\|z\|^{2}-2\langle z, g(u)\rangle+\|g(u)\|^{2}\right) .
$$

It is easy to see that $\phi(\cdot, u)$ is Lipschitz continuous with modulus being bounded by $\sup _{z \in \mathcal{Y}, u \in \mathcal{U}}(\|z\|+$ $\|g(u)\|)$, where $\mathcal{U}=B\left(\bar{u}, \bar{\delta}_{u}\right) \cdot \phi(z, \cdot)$ is Hölder continuous at $\bar{u}$, that is,

$$
|\phi(z, u)-\phi(z, \bar{u})| \leq \varrho \sup _{z \in \mathcal{Y}, u \in \mathcal{U}}(\|z\|+\|g(u)\|)\|u-\bar{u}\|_{U}^{\gamma}
$$

when $u \in B\left(\bar{u}, \bar{\delta}_{u}\right)$. This verifies conditions (a) and (b) of Theorem 2.1. To see the growth condition, the orthogonal project of $g(u)$ over the convex set $\Psi(\bar{u})$ means that

$$
\left\langle\Pi_{\Psi(\bar{u})}(g(\bar{u}))-g(\bar{u}), z-\Pi_{\Psi(\bar{u})}(g(\bar{u}))\right\rangle \geq 0, \forall z \in \Psi(\bar{u}) .
$$

With the inequality, we can obtain

$$
\begin{aligned}
\phi(z, \bar{u})-\vartheta(\bar{u}) & =\frac{1}{2}\|z-g(\bar{u})\|^{2}-\frac{1}{2}\left\|\Pi_{\Psi(\bar{u})}(g(\bar{u}))-g(\bar{u})\right\|^{2} \\
& \geq \frac{1}{2}\left\|z-\Pi_{\Psi(\bar{u})}(g(\bar{u}))\right\|^{2}
\end{aligned}
$$

Note that the second order growth condition holds when $\bar{u}$ is replaced by any $u$. The proof is complete. 


\subsection{Pseudometric}

Let $F(x, y, \xi)$ and $G(x, y, z, \xi)$ be defined as in (1.1) and (1.2). Let $\mathscr{B}$ denote the $\sigma$-algebra of all Borel subsets of $\Xi$ (the support set of $\xi$ ) and $\mathscr{P}$ be the set of all probability measures of the measurable space $(\Xi, \mathscr{B})$ induced by $\xi$. For compact sets $\mathcal{X} \subseteq \mathbb{R}^{n}$ and $\mathcal{Y} \subseteq \mathbb{R}^{m}$, let

$$
\begin{gathered}
\mathscr{F}:=\{F(x, y, \cdot): x \in \mathcal{X}, y \in \mathcal{Y}\} \\
\mathscr{G}:=\{G(x, y, z, \cdot): x \in \mathcal{X}, y \in \mathcal{Y}, z \in \mathcal{Y}\} .
\end{gathered}
$$

For any probability measures $P, Q \in \mathscr{P}$ (here we are slightly abusing the notation as $P$ and $Q$ refer to probability measures of the measurable space $(\Omega, \mathcal{F})$ in the introduction, a correct reading/interpretation is that $P$ and $Q$ here are two different distributions of $\xi$ whose support set is contained in $\Xi$ and we use the terminology probability measure and probability distribution interchangeably), define the distances

$$
\begin{aligned}
\mathscr{D}_{F}(Q, P) & :=\sup _{f \in \mathscr{F}}\left\|\mathbb{E}_{P}[f(\xi)]-\mathbb{E}_{Q}[f(\xi)]\right\|, \\
\mathscr{D}_{G}(Q, P) & :=\sup _{g \in \mathscr{G}}\left\|\mathbb{E}_{P}[g(\xi)]-\mathbb{E}_{Q}[g(\xi)]\right\|_{W},
\end{aligned}
$$

and

$$
\mathscr{D}_{H}(Q, P):=\max \left\{\mathscr{D}_{F}(Q, P), \mathscr{D}_{G}(Q, P)\right\} .
$$

It is easy to see that $\mathscr{D}_{F}(Q, P)=0$ if and only if $\mathbb{E}_{P}[f(\xi)]=\mathbb{E}_{Q}[f(\xi)]$ for all $f \in \mathscr{F}$ but it does not necessarily mean $P=Q$ unless the set $\mathscr{F}$ is sufficiently large. Similar comments apply to $\mathscr{D} G(Q, P)$. This type of distance is widely used for stability analysis of stochastic programming and is known as pseudometric in that it satisfies all properties of a metric except that $\mathscr{D}_{F}(Q, P)=0$ does not necessarily imply $P=Q$ unless the set of functions $\mathscr{F}$ is sufficiently large. For a comprehensive discussion of the concept of pseudometric and related issues, see [33].

It might be interesting to give a couple of examples where a perturbation $Q$ approximates $P$ under the pseudometric. One is empirical probability measure defined as

$$
P_{N}(\cdot):=\frac{1}{N} \sum_{k=1}^{N} \mathbb{1}_{\xi^{k}}(\cdot)
$$

where $\xi^{1}, \cdots, \xi^{N}$ are independent and identically distributed random variables defined on $(\Omega, \mathcal{F})$ having the same distribution as $\xi, \mathbb{1}_{\xi^{k}}(\cdot)$ is an indicator function taking value 1 when $\xi=\xi^{k}$ and 0 otherwise. By the well known Glivenko-Cantelli theorem [38], $P_{N}$ converges to $P$ weakly as $N$ goes to infinity. Since $\mathcal{X}$ and $\mathcal{Y}$ are compact, $F$ and $G$ are continuous w.r.t. $\xi$, then the classes of functions $\mathscr{F}$ and $\mathscr{G}$ defined in Section 2.4 are uniformly bounded. This ensures $\mathscr{D}_{H}\left(P_{N}, P\right)$ converges to zero as $N$ tends to infinity hence Theorem 4.1 in Section 4 can be applied to sample average approximation of problem (1.1).

Another example is when $Q$ is a probability measure which approximates $P$ under Kantorovich metric, Wasserstein metric or bounded Lipschitz metric in that these metrics metrize weak convergence, see [24].

Corollary 2.1 and pseudo metric $\mathscr{D}_{H}(Q, P)$ pave the way for us to quantify the gap between $\Pi_{\Gamma_{Q}(x, y)}\left(y-\mathbb{E}_{Q}[F(x, y, \xi)]\right)$ and $\Pi_{\Gamma_{P}\left(x_{0}, y\right)}\left(y-\mathbb{E}_{P}\left[F\left(x_{0}, y, \xi\right)\right]\right)$ in Section 4. 


\section{Existence of a solution to the perturbed SQVIP}

In this section, we discuss existence of solutions to the perturbed SQVIP (1.6) before we move on to stability analysis in the next section. To this end, we assume that $\mathcal{Y}$ is a compact set throughout this section and make the following assumptions.

Assumption 3.1 Consider (1.2). There exists $x_{0} \in \mathbb{R}^{n}$ such that

$$
0 \in \operatorname{int}\left\{\mathbb{E}_{P}\left[G\left(x_{0}, y, \mathcal{Y}, \xi\right)\right]-\mathcal{K}\right\}, \quad \forall y \in \mathcal{Y},
$$

where $\mathbb{E}_{P}\left[G\left(x_{0}, y, \mathcal{Y}, \xi\right)\right]:=\left\{\mathbb{E}_{P}\left[G\left(x_{0}, y, z, \xi\right)\right]: z \in \mathcal{Y}\right\}$.

Condition (3.1) is a Slater constraint qualification corresponding to the one we use in Section 2.3. Note that the condition implicitly assumes $\Gamma_{P}\left(x_{0}, y\right) \neq \emptyset$ for any $y \in \mathcal{Y}$.

Assumption 3.2 Let $x_{0}$ be defined as in Assumption 3.1 and $\hat{\mathcal{P}} \subset \mathscr{P}$ be a set of probability measures such that $P, Q \in \hat{\mathcal{P}}$. Let $G(x, y, z, \xi)$ be defined as in (1.2). The following hold.

(a) For each fixed $\xi \in \Xi, G(x, y, z, \xi)$ is uniformly locally Lipschitz continuous in $x$ at $x_{0}$ and globally Lipschitz continuous in $z$ on $\mathcal{Y}$ with Lipschitz modulus being bounded by $\kappa(\xi)$, where $\sup _{P \in \hat{\mathcal{P}}} \mathbb{E}_{P}[\kappa(\xi)]<\infty$.

(b) For each fixed $(y, z) \in \mathcal{Y} \times \mathcal{Y}$, $\sup _{P \in \hat{\mathcal{P}}}\left\|\mathbb{E}_{P}\left[G\left(x_{0}, y, z, \xi\right)\right]\right\|_{W}<\infty$.

(c) For each fixed $\xi \in \Xi, F(x, y, \xi)$ is uniformly locally Lipschitz continuous in $x$ at $x_{0}$ with Lipschitz modulus being bounded by $\kappa(\xi)$ and continuous in $y$ on $\mathcal{Y}$, where $\sup _{P \in \hat{\mathcal{P}}} \mathbb{E}_{P}[\kappa(\xi)]<\infty$.

(d) For each fixed $y \in \mathcal{Y}$, $\sup _{P \in \hat{\mathcal{P}}}\left\|\mathbb{E}_{P}\left[F\left(x_{0}, y, \xi\right)\right]\right\|<\infty$.

Assumption $3.2(\mathrm{~b})$ ensures well definedness of $\mathbb{E}_{Q}\left[G\left(x_{0}, y, z, \xi\right)\right]$ for each $(y, z) \in \mathcal{Y} \times \mathcal{Y}$. Together with Assumption 3.2 (a), it also guarantees well definedness of $\mathbb{E}_{Q}[G(x, y, z, \xi)]$ for $x$ near $x_{0}$. Moreover, through Lebesgue dominance convergence theorem, $\mathbb{E}_{Q}[G(x, y, \cdot, \xi)]$ is continuous on $\mathcal{Y}$ for each fixed $Q$ close to $P$ and $y \in \mathcal{Y}$. Similar comments apply to $\mathbb{E}_{Q}\left[G\left(x_{0}, y, z, \xi\right)\right]$ under Assumptions 3.2 (c) and (d). With Assumptions 3.1 and 3.2, we can easily obtain the following.

Proposition 3.1 Let Assumptions 3.1 and 3.2 hold and $x_{0} \in \mathbb{R}^{n}$ be given as in Assumption 3.1. Then the following assertions hold.

(i) Both $\mathbb{E}_{Q}[G(x, y, z, \xi)]$ and $\mathbb{E}_{Q}[F(x, y, \xi)]$ are uniformly Lipschitz continuous in $Q$ (independent of $x$ and $y$ ) and uniformly continuous in $x$ at $x_{0}$ (independent of $Q$ and $y$ ), that is, there exists a positive constant $\bar{\delta}_{x}$ such that

$$
\left\|\mathbb{E}_{Q}[G(x, y, z, \xi)]-\mathbb{E}_{P}\left[G\left(x_{0}, y, z, \xi\right)\right]\right\|_{W} \leq \mathscr{D}_{G}(P, Q)+\sup _{P \in \hat{\mathcal{P}}} \mathbb{E}_{P}[\kappa(\xi)]\left\|x-x_{0}\right\|
$$

and

$$
\left\|\mathbb{E}_{Q}[F(x, y, \xi)]-\mathbb{E}_{P}\left[F\left(x_{0}, y, \xi\right)\right]\right\| \leq \mathscr{D}_{F}(P, Q)+\sup _{P \in \hat{\mathcal{P}}} \mathbb{E}_{P}[\kappa(\xi)]\left\|x-x_{0}\right\|
$$

for any $(y, z) \in \mathcal{Y} \times \mathcal{Y}$ and $x \in B_{n}\left(x_{0}, \bar{\delta}_{x}\right) \cap \mathcal{X}$. 
(ii) There exists a positive constant $\bar{\delta}_{P, x} \leq \bar{\delta}_{x}$ such that the following Slater Constraint Qualification holds:

$$
0 \in \operatorname{int}\left\{\mathbb{E}_{Q}[G(x, y, \mathcal{Z}, \xi)]-\mathcal{K}\right\}, \quad \forall y \in \mathcal{Y},
$$

when $Q \in U\left(P, \bar{\delta}_{P, x}\right)$ and $x \in B_{n}\left(x_{0}, \bar{\delta}_{P, x}\right) \cap \mathcal{X}$. Here and later on, $U(P, \delta):=\{Q \in \mathscr{P}$ : $\left.\mathscr{D}_{H}(Q, P)<\delta\right\}$ for a positive constant $\delta$.

(iii) $\Gamma_{Q}(x, y)$ is nonempty for $Q \in U\left(P, \bar{\delta}_{P, x}\right)$ and $x \in B_{n}\left(x_{0}, \bar{\delta}_{P, x}\right) \cap \mathcal{X}$, and $\Gamma_{Q}(x, \cdot)$ is continuous over $\mathcal{Y}$ for $x \in B_{n}\left(x_{0}, \bar{\delta}_{P, x}\right) \cap \mathcal{X}$.

(iv) For any $y_{0} \in \mathcal{Y}$ and $x \in B_{n}\left(x_{0}, \bar{\delta}_{P, x}\right) \cap \mathcal{X}$,

$$
\lim _{y \rightarrow y_{0}} \Pi_{\Gamma_{P}(x, y)}(z)=\Pi_{\Gamma_{P}\left(x, y_{0}\right)}(z) .
$$

Proof. Part (i). Let $\mathcal{X}$ be a compact neighborhood of $x_{0}$. Observe first that for each fixed $x \in \mathcal{X}$ and $y \in \mathcal{Y}, \mathbb{E}_{P}[G(x, y, z, \xi)]$ is continuous w.r.t. variation of probability measure $P$ under the pseudometric $\mathscr{D}$ defined in Section 2. In fact, for any $P, Q \in \hat{\mathcal{P}}$

$$
\left\|\mathbb{E}_{P}\left[G\left(x_{0}, y, z, \xi\right)\right]-\mathbb{E}_{Q}\left[G\left(x_{0}, y, z, \xi\right)\right]\right\|_{W} \leq \mathscr{D}_{G}(P, Q)
$$

for any $(y, z) \in \mathcal{Y} \times \mathcal{Y}$. Under Assumption 3.2 and the compactness of $\mathcal{X}$, we have

$$
\sup _{x \in \mathcal{X}, P \in \hat{\mathcal{P}}}\left\|\mathbb{E}_{P}[G(x, y, z, \xi)]\right\|_{W}<\infty
$$

for any $(y, z) \in \mathcal{Y} \times \mathcal{Y}$. On the other hand, for any $x \in B_{n}\left(x_{0}, \bar{\delta}_{P, x}\right) \cap \mathcal{X}$,

$$
\left\|\mathbb{E}_{Q}[G(x, y, z, \xi)]-\mathbb{E}_{Q}\left[G\left(x_{0}, y, z, \xi\right)\right]\right\|_{W} \leq \sup _{Q \in \hat{\mathcal{P}}} \mathbb{E}_{Q}[\kappa(\xi)]\left\|x-x_{0}\right\|
$$

for any $(y, z) \in \mathcal{Y} \times \mathcal{Y}$. Combining (3.5) and (3.6), we obtain (3.2). We obtain (3.3) in the same way.

Part (ii). For any $\epsilon>0$, there exists positive constant $\bar{\delta}_{P, x}^{\prime}<\bar{\delta}_{x}$ such that

$$
-\mathbb{E}_{P}\left[G\left(x_{0}, y, z, \xi\right)\right]+\mathcal{K} \subset-\mathbb{E}_{Q}[G(x, y, z, \xi)]+\mathcal{K}+\epsilon \mathcal{B}_{W}
$$

for all $(y, z) \in \mathcal{Y} \times \mathcal{Y}$ when $\max \left\{\mathscr{D}_{G}(P, Q),\left\|x-x_{0}\right\|\right\} \leq \bar{\delta}_{P, x}^{\prime}$, where $\mathcal{B}_{W}$ denotes the unit ball in the space $W$ equipped with norm $\|\cdot\|_{W}$, that is, $\mathcal{B}_{W}:=\left\{v \in W:\|v\|_{W} \leq 1\right\}$. This shows

$$
-\mathbb{E}_{P}\left[G\left(x_{0}, y, \mathcal{Y}, \xi\right)\right]+\mathcal{K} \subset-\mathbb{E}_{Q}[G(x, y, \mathcal{Y}, \xi)]+\mathcal{K}+\epsilon \mathcal{B}_{W}
$$

By Assumption 3.1, there exists $\tau>0$ such that

$$
\tau \mathcal{B}_{W} \subseteq-\mathbb{E}_{P}\left[G\left(x_{0}, y, \mathcal{Y}, \xi\right)\right]+\mathcal{K}
$$

Let $\epsilon:=\tau / 2$. By (3.7), there exists positive constant $\bar{\delta}_{P, x}<\bar{\delta}_{P, x}^{\prime}$ such that

$$
\frac{\tau}{2} \mathcal{B}_{W} \subseteq-\mathbb{E}_{Q}[G(x, y, \mathcal{Y}, \xi)]+\mathcal{K}
$$

for any $y \in \mathcal{Y}$ when $\max \left\{\mathscr{D}_{G}(P, Q),\left\|x-x_{0}\right\|\right\} \leq \bar{\delta}_{P, x}$. 
Part (iii). The nonemptiness comes from Part (ii). We make use of Lemma 2.2 (i) to show the continuity by treating $\left(\mathbb{E}_{Q}[\cdot], x, y\right)$ as a parameter. The convexity of $\mathbb{E}_{Q}[G(x, y, z, \xi)]$ in $z$ is obvious under our generic assumption that $G$ is convex in $z$ for each $x, y, \xi$ in the definition of SQVIP in Section 1. Part (i) of this proposition addresses condition (b) of the lemma whereas the Slater condition is explicitly assumed here. Therefore all conditions for Lemma 2.2 (i) are verified.

Part (iv). The conclusion follows by Part (ii) and [26, Proposition 4.9].

Proposition 3.1 paves the way for us to state our main result in this section which asserts existence of solutions to the perturbed SQVIP (1.6).

Theorem 3.1 Under Assumptions 3.1-3.2, $S_{Q}(x)$ is a nonempty and compact set under pseudometric $\mathscr{D}$ for $Q \in U\left(P, \bar{\delta}_{P, x}\right)$ and $x \in B\left(x_{0}, \bar{\delta}_{P, x}\right)$.

Proof. We prove the result by making use of [22, Theorem 1]. It therefore suffices to verify the conditions of the theorem. From Proposition 3.1, we know that for fixed $Q$ close to $P$ and $x$ close to $x_{0}, \Gamma_{Q}(x, y)$ is a nonempty convex set for any $y \in \mathcal{Y}$ and $\Gamma_{Q}(x, \cdot)$ is continuous on $\mathcal{Y}$. The closedness of $\Gamma_{Q}(x, y)$ is due to continuity of $\mathbb{E}_{Q}[G(x, y, \cdot, \xi)]$ for fixed $x$ and $y$ and closedness of $\mathcal{K}$.

In the literature of deterministic QVIP, conditions for existence have been well investigated. For instance, under compactness assumptions, Pang and Fukushima [22] established an existence result without assuming any special structure of $\Gamma_{P}(x, y)$. Existence results without compactness condition are obtained by Facchinei and Pang [11]. Our Theorem 3.1 may be regarded as an analogue of [22, Theorem 1] for the stochastic QVIP (1.6).

Note that Assumptions 3.1 is necessary to ensure the nonemptiness of $S_{Q}(x)$. The following example shows that the set of solutions may be empty when the condition fails.

Example 3.1 Consider the following SQVIP

$$
0 \in x^{2}+\mathbb{E}_{P}\left[e^{y \xi}\right]+\mathcal{N}_{\Gamma_{P}(x, y)}(y), x, y \in \mathbb{R},
$$

where $x \in[-e-0.5, e+0.5]$ is a parameter, $e$ is the Euler number, $y \in \mathcal{Y}:=[-1,1]$ is a decision variable, $\xi$ is a random variable continuously distributed over set $[-1,1]$ with distribution $P$ and

$$
\Gamma_{P}(x, y):=\left\{z \in[-1,1]:\left(x^{2}-\mathbb{E}_{P}\left[e^{y \xi}\right] y\right) z \geq 0\right\} .
$$

Let $x_{0}=0$ be fixed. We show that the SCQ (3.1) fails at $x_{0}$. Observe that $G(x, y, z, \xi)=\left(x^{2}-e^{y \xi} y\right) z$, $\mathbb{E}_{P}\left[G\left(x_{0}, y, \mathcal{Y}, \xi\right)\right]=\left(x_{0}^{2}-\mathbb{E}_{P}\left[e^{y \xi}\right] y\right)[-1,1]$ and $\mathcal{K}=\mathbb{R}_{+}$. For $y_{0}=0 \in \mathcal{Y}, \mathbb{E}_{P}\left[G\left(x_{0}, y_{0}, \mathcal{Y}, \xi\right)\right]=$ $\left(x_{0}^{2}-\mathbb{E}_{P}\left[e^{y_{0} \xi}\right] y_{0}\right)[-1,1]=0$. Thus

$$
0 \notin \operatorname{int}\left\{0-\mathbb{R}_{+}\right\}=\operatorname{int}\left\{\mathbb{E}_{P}\left[G\left(x_{0}, y_{0}, \mathcal{Y}, \xi\right)\right]-\mathcal{K}\right\} .
$$

Let $\tilde{S}(x)$ denote the set of solutions to (3.9). In what follows, we show that $\tilde{S}\left(x_{0}\right)=\emptyset$. It is easy to verify that for $(x, y) \in[-e-0.5, e+0.5] \times[-1,1]$,

$$
\Gamma_{P}(x, y)= \begin{cases}{[0,1],} & \text { for } x^{2}>\mathbb{E}_{P}\left[e^{y \xi}\right] y, \\ {[-1,1],} & \text { for } x^{2}=\mathbb{E}_{P}\left[e^{y \xi}\right] y, \\ {[-1,0],} & \text { for } x^{2}<\mathbb{E}_{P}\left[e^{y \xi}\right] y\end{cases}
$$


By the definition of the normal cone in convex analysis, we have for $(x, y) \in[-e-0.5, e+0.5] \times[-1,1]$,

$$
\begin{aligned}
& \mathcal{N}_{\Gamma_{P}(x, y)}(y)=\left\{\begin{array}{cl}
\mathbb{R}_{-}, & \text {for } x^{2}>\mathbb{E}_{P}\left[e^{y \xi}\right] y, y=0, \\
\{0\}, & \text { for } x^{2}>\mathbb{E}_{P}\left[e^{y \xi}\right] y, y \in(0,1), \\
\mathbb{R}_{+}, & \text {for } x^{2}>\mathbb{E}_{P}\left[e^{y \xi}\right] y, y=1, \\
\emptyset, & \text { for } x^{2}>\mathbb{E}_{P}\left[e^{y \xi} y, y \in[-1,0),\right. \\
\{0\}, & \text { for } x^{2}=\mathbb{E}_{P}\left[e^{y \xi}\right] y, y \in[0,1), \\
\mathbb{R}_{+}, & \text {for } x^{2}=\mathbb{E}_{P}\left[e^{y \xi}\right] y, y=1, \\
\emptyset, & \text { for } x^{2}=\mathbb{E}_{P}\left[e^{y \xi}\right] y, y \in[-1,0), \\
\emptyset, & \text { for } x^{2}<\mathbb{E}_{P}\left[e^{y \xi}\right] y, y \in[-1,1] .
\end{array}\right. \\
&=\left\{\begin{array}{cl}
\mathbb{R}_{-}, & \text {for } x^{2}>\mathbb{E}_{P}\left[e^{y \xi}\right] y, y=0, \\
\{0\}, & \text { for } x^{2}>\mathbb{E}_{P}\left[e^{y \xi} y, y \in(0,1) \text { or } x^{2}=\mathbb{E}_{P}\left[e^{y \xi}\right] y, y \in[0,1),\right. \\
\mathbb{R}_{+}, & \text {for } x^{2}>\mathbb{E}_{P}\left[e^{y \xi}\right] y, y=1 \text { or } x^{2}=\mathbb{E}_{P}\left[e^{y \xi}\right] y, y=1, \\
\emptyset, & \text { otherwise. }
\end{array}\right.
\end{aligned}
$$

Since $x_{0}=0$,

$$
\mathcal{N}_{\Gamma_{P}\left(x_{0}, y\right)}(y)= \begin{cases}\{0\}, & \text { for } y=0 \\ \emptyset, & \text { otherwise }\end{cases}
$$

hence

$$
\tilde{S}\left(x_{0}\right)=\left\{y \in[-1,1]: 0 \in \mathbb{E}_{P}\left[e^{y \xi}\right]+\mathcal{N}_{\Gamma_{P}(0, y)}(y)\right\}=\emptyset .
$$

However, the SCQ holds at $x_{0} \in[e-0.5, e+0.5]$ and $\tilde{S}\left(x_{0}\right) \neq \emptyset$ in this case.

\section{Stability of SQVIP}

In this section, we return to the main topic of the paper, that is, quantitative stability analysis of SQVIP (1.1). Specifically we investigate the relationship between the set of solutions to the perturbed SQVIP (1.6) and its true counterpart (1.1) when parameter $x$ varies locally at certain fixed point $x_{0}$ and the probability measure $Q$ varies locally at $P$ under the pseudometric $\mathscr{D}_{H}(Q, P)$ defined in Section 2.3. As explained in the introduction, we plan to do so through the normal equations (1.8) and (1.5). A key step is to estimate the discrepancy between $\mathbf{F}_{Q}^{\text {nat }}(x, y)$ and $\mathbf{F}_{P}^{\text {nat }}\left(x_{0}, y\right)$ which can be quantified by

$$
\left\|\Pi_{\Gamma_{Q}(x, y)}\left(y-\mathbb{E}_{Q}[F(x, y, \xi)]\right)-\Pi_{\Gamma_{P}(x, y)}\left(y-\mathbb{E}_{P}\left[F\left(x_{0}, y, \xi\right)\right]\right)\right\| .
$$

Observe that for fixed $(x, y) \in \mathbb{R}^{n} \times \mathbb{R}^{m}$ and $Q \in \hat{\mathcal{P}}$,

$\Pi_{\Gamma_{Q}(x, y)}\left(y-\mathbb{E}_{Q}[F(x, y, \xi)]\right)$ is the unique optimal solution to the following convex minimization problem

$$
\begin{array}{ll}
\min _{z \in \mathcal{Y}} & \frac{1}{2}\left\|z-y+\mathbb{E}_{Q}[F(x, y, \xi)]\right\|^{2} \\
\text { s.t. } & \mathbb{E}_{Q}[G(x, y, z, \xi)] \in \mathcal{K},
\end{array}
$$

where $z$ is the only decision vector, whereas $x$ and $y$ are parameters. In other words, the quantity to be estimated in (4.11) is essentially the deviation of the optimal solution of problem (4.12) from its true counterpart if we regard $Q$ and $x$ as a perturbation from $P$ and $x_{0}$. 
Along this line, we may carry out stability analysis of SQVIP (1.1) through problem (4.12). On the other hand, the latter may be treated as a special case of parametric minimization problem (2.11) and this is indeed one of the underlying reasons for us to present the stability analysis for generic parametric minimization problems in Section 2. To see this argument clearly, let $u=\left(\mathbb{E}_{Q}[\cdot, \cdot], x, y\right)$. The only thing we need to explain is that the mathematical expectation operation $\mathbb{E}_{Q}[\cdot]$ can indeed be treated as a parameter in the metric space of probability measure $\mathscr{P}$. Let $\mathscr{D}_{H}(Q, P) \leq \delta$. For any nonnegative measure $\mu$ defined over $\Omega$, let

$$
\langle\mu, h\rangle:=\int_{\Omega} h(\xi(\omega)) \mu(d \omega) .
$$

Then $\mathbb{E}_{Q}[F(x, y, \xi)]$ may be written as $\left\langle\mathbb{E}_{Q}, F(x, y, \xi)\right\rangle$. In doing so, we can effectively treat operation $\mathbb{E}_{Q}[\cdot]$ as a parameter in the space of probability measures $\mathscr{P}$ equipped with the pseudometric.

Using Corollary 2.1, we are able to present quantitative stability analysis for problem (4.12) which is one of the main technical results in this section.

Proposition 4.1 Let Assumptions 3.1-3.2 hold. Then there exist positive constants $\rho, \bar{\delta}$ and $\beta \in(0,1)$ such that

$$
\left\|\mathbf{F}_{Q}^{\text {nat }}(x, y)-\mathbf{F}_{P}^{\text {nat }}\left(x_{0}, y\right)\right\| \leq \rho\left(\mathscr{D}_{H}(Q, P)+\left\|x-x_{0}\right\|\right)^{\beta}
$$

for any $y \in \mathcal{Y}, x \in B_{n}\left(x_{0}, \bar{\delta}\right) \cap \mathcal{X}$ and $Q \in U(P, \bar{\delta})$, where $U(P, \bar{\delta})$ is defined as in Proposition 3.1.

Proof. We use Corollary 2.1 to prove the result. Note that the corollary has two set of conditions: one for the objective function while the other for the constraints.

Let $g(u):=\mathbb{E}_{Q}[y-F(x, y, \xi)]$ and $\psi(z, u):=\mathbb{E}_{Q}[G(x, y, z, \xi)]$ where $u$ is a parameter comprising three components: $x, y$ and $\mathbb{E}_{Q}[\cdot]$. Let $g(\bar{u}):=\mathbb{E}_{P}\left[y-F\left(x_{0}, y, \xi\right)\right]$ and $\psi(z, \bar{u}):=\mathbb{E}_{P}\left[G\left(x_{0}, y, z, \xi\right)\right]$ with $\bar{u}$ representing $x_{0}, y$ and $\mathbb{E}_{P}[\cdot]$.

Conditions concerning the constraints are essentially about $-\mathcal{K}$-convexity of $\psi(\cdot, u)$, uniform Hölder continuity $\psi$ in $u$ and Slater condition. In this context, convexity is straightforward, while the other two properties are either addressed in Proposition 3.1 (i) or explicitly assumed.

The condition concerning the objective function is even simpler because $g(u)$ is indeed Lipschitz continuous by Proposition 3.1 (i).

With Proposition 4.1, we are ready to state the main stability result of this section.

Theorem 4.1 Let $\mathcal{X} \subseteq \mathbb{R}^{n}$ and $\mathcal{Y} \subseteq \mathbb{R}^{m}$ be compact sets. Under Assumptions 3.1-3.2, the following assertions hold.

(i) There exist positive constants $\delta, \rho$ and $\beta \in(0,1)$ such that

$$
\mathbb{D}\left(S_{Q}(x), S_{P}\left(x_{0}\right)\right) \leq \Upsilon\left(2 \rho\left(\mathscr{D}_{H}(Q, P)+\left\|x-x_{0}\right\|\right)^{\beta}\right)
$$

for any $(x, Q) \in B_{n}\left(x_{0}, \delta\right) \cap \mathcal{X} \times U(P, \delta)$, where for a small positive number $\varepsilon$,

$$
R(\varepsilon)=\inf _{y \in \mathcal{Y}, d\left(y, S_{P}\left(x_{0}\right)\right) \geq \varepsilon}\left\|\mathbf{F}_{P}^{\text {nat }}\left(x_{0}, y\right)\right\|,
$$

$\Upsilon(t)=\min \left\{\varepsilon \in \mathbb{R}_{+}: R(\varepsilon)=t\right\}$ and $\Upsilon(t) \rightarrow 0$ as $t \downarrow 0$. 
(ii) If $\mathbf{F}_{P}^{\text {nat }}\left(x_{0}, y\right)$ is metrically regular at each $y^{*} \in S_{P}\left(x_{0}\right)$ for 0 , then there exist positive constants $c$ and $\delta$ such that

$$
\mathbb{D}\left(S_{Q}(x), S_{P}\left(x_{0}\right)\right) \leq c\left(\mathscr{D}_{H}(Q, P)+\left\|x-x_{0}\right\|\right)^{\beta}
$$

for any $(x, Q) \in B_{n}\left(x_{0}, \delta\right) \cap \mathcal{X} \times U(P, \delta)$.

(iii) If $\mathbf{F}_{P}^{\text {nat }}\left(x_{0}, y\right)$ is a locally Lipschitz homeomorphism at $y^{*} \in S_{P}\left(x_{0}\right)$, then there exist positive constants $c, \delta$ and a neighborhood $\mathcal{V}$ of $y^{*}$ such that

$$
\left\|y-y^{*}\right\| \leq c\left(\mathscr{D}_{H}(Q, P)+\left\|x-x_{0}\right\|\right)^{\beta}
$$

for any $y \in S_{Q}(x) \cap \mathcal{V}$ with $(x, Q) \in B_{n}\left(x_{0}, \delta\right) \cap \mathcal{X} \times U(P, \delta)$.

Proof. Part (i). First, by the definition of $R(\varepsilon), R(0)=0$ and $R(\varepsilon)$ is nondecreasing on $[0,+\infty)$. We show that $R(\varepsilon)>0$ for $\varepsilon>0$. Assume for the sake of a contradiction that $R(\varepsilon)=0$ for some $\varepsilon>0$. Then there exists a sequence $\left\{y_{k}\right\} \subseteq \mathcal{Y}$ such that $d\left(y_{k}, S_{P}\left(x_{0}\right)\right) \geq \varepsilon$ and

$$
\lim _{k \rightarrow \infty}\left\|\mathbf{F}_{P}^{\text {nat }}\left(x_{0}, y_{k}\right)\right\|=0 \text {. }
$$

Since $\mathcal{Y}$ is a compact set, we may assume without loss of generality that $y_{k} \rightarrow \bar{y}$ as $k \rightarrow \infty$. Then $\bar{y} \in \mathcal{Y}$ and by Proposition 3.1 (iv)

$$
\lim _{k \rightarrow \infty}\left\|\mathbf{F}_{P}^{\text {nat }}\left(x_{0}, y_{k}\right)-\mathbf{F}_{P}^{\text {nat }}\left(x_{0}, \bar{y}\right)\right\|=0
$$

which, together with (4.17), yield $\mathbf{F}_{P}^{\text {nat }}\left(x_{0}, \bar{y}\right)=0$ and hence $\bar{y} \in S_{P}\left(x_{0}\right)$. This leads to a contradiction as desired because $d\left(\bar{y}, S_{P}\left(x_{0}\right)\right) \geq \varepsilon>0$. The discussion manifests that $\Upsilon(t) \rightarrow 0$ as $t \downarrow 0$.

Second, there exists a positive number $t_{0}$ such that $\Upsilon(t)$ is well defined over interval $\left[0, t_{0}\right]$. To this end, we show $R(\varepsilon)$ is continuous over interval $\left[0, \varepsilon_{0}\right]$ with $t_{0}=R\left(\varepsilon_{0}\right)$.

Since $R(\varepsilon)>0$ for $\varepsilon>0$, there exist $\varepsilon_{0}>0$ and $t_{0}>0$ with $R\left(\varepsilon_{0}\right)=t_{0}$. Let

$$
\mathcal{F}(\varepsilon):=\left\{y \in \mathcal{Y}: d\left(y, S_{P}\left(x_{0}\right)\right) \geq \varepsilon\right\} .
$$

Then $\mathcal{F}\left(\varepsilon_{1}\right) \subseteq \mathcal{F}\left(\varepsilon_{2}\right)$ for $\varepsilon_{2}<\varepsilon_{1}$ and $\varepsilon_{1}, \varepsilon_{2} \in\left[0, \varepsilon_{0}\right]$. This means that $\mathcal{F}(\varepsilon) \neq \emptyset$ for $\varepsilon \in\left[0, \varepsilon_{0}\right]$ due to the fact that $\mathcal{F}\left(\varepsilon_{0}\right) \neq \emptyset$. By [26, Exercise 4.3],

$$
\lim _{\varepsilon \rightarrow \varepsilon^{\prime}} \mathcal{F}(\varepsilon)=\mathcal{F}\left(\varepsilon^{\prime}\right)
$$

for $\varepsilon^{\prime} \in\left[0, \varepsilon_{0}\right]$. On the other hand, $R(\varepsilon)$ may be viewed as the optimal value of the minimization problem parameterized by $\varepsilon$ with objective function $\left\|\mathbf{F}_{P}^{\text {nat }}\left(x_{0}, y\right)\right\|$ and feasible set $\mathcal{F}(\varepsilon)$. By the classical stability theorem of parametric optimization problem (see e.g. [17, Theorem 1.15]), we know that $R(\varepsilon)$ is continuous on $\left[0, \varepsilon_{0}\right]$. Thus by the Mean Value Theorem, for any $t \in\left[0, t_{0}\right]$, there exists $\varepsilon \in\left[0, \varepsilon_{0}\right]$ such that $R(\varepsilon)=t$, which means that $\Upsilon(t)$ is well defined for any $t \in\left[0, t_{0}\right]$.

In what follows, we show (4.14). Let $\varepsilon$ be a fixed small positive number and $y \in \mathcal{Y}$ with $d\left(y, S_{P}\left(x_{0}\right)\right)>\varepsilon$. Then $\left\|\mathbf{F}_{P}^{\text {nat }}\left(x_{0}, y\right)\right\| \geq R(\varepsilon)$. Moreover, by Proposition 4.1 , there exist constants $\bar{\delta}>0, \rho>0$ and $\beta \in(0,1)$ such that

$$
\begin{aligned}
\left\|\mathbf{F}_{Q}^{\text {nat }}(x, y)\right\| & \geq\left\|\mathbf{F}_{P}^{\text {nat }}\left(x_{0}, y\right)\right\|-\left\|\mathbf{F}_{Q}^{\text {nat }}(x, y)-\mathbf{F}_{P}^{\text {nat }}\left(x_{0}, y\right)\right\| \\
& \geq R(\varepsilon)-\rho\left(\mathscr{D}_{H}(Q, P)+\left\|x-x_{0}\right\|\right)^{\beta}
\end{aligned}
$$


for any $Q \in \mathscr{P}$ with $\mathscr{D}_{H}(Q, P) \leq \bar{\delta}$ and $x \in \mathcal{X}$ with $\left\|x-x_{0}\right\|<\bar{\delta}$. Let $\bar{\delta}$ be sufficiently small such that

$$
\rho\left(\mathscr{D}_{H}(Q, P)+\left\|x-x_{0}\right\|\right)^{\beta} \leq R(\varepsilon) / 2 .
$$

Then we arrive at $\left\|\mathbf{F}_{Q}^{\text {nat }}(x, y)\right\| \geq R(\varepsilon) / 2>0$ which implies $y \notin S_{Q}(x)$. This shows

$$
\mathbb{D}\left(S_{Q}(x), S_{P}\left(x_{0}\right)\right) \leq \varepsilon
$$

for any $Q \in \mathscr{P}$ with $\mathscr{D}_{H}(Q, P)<\bar{\delta}$ and $x \in \mathcal{X}$ satisfying $\left\|x-x_{0}\right\|<\bar{\delta}$. Let $\varepsilon$ be the minimal value such that

$$
R(\varepsilon) / 2=\rho\left(\mathscr{D}_{H}(Q, P)+\left\|x-x_{0}\right\|\right)^{\beta} .
$$

The rest follows from the definition of $\Upsilon$.

Part (ii). Since $\mathbf{F}_{P}^{\text {nat }}\left(x_{0}, y\right)$ is metrically regular at $y^{*}$ for 0 with modulus $\alpha>0$ and from Part (i) $S_{Q}(x)$ is upper semicontinuous w.r.t. $(Q, x)$ at $\left(P, x_{0}\right)$, there exist positive constants $\delta_{1}, \delta_{2}, \delta_{3}$ and $\alpha$ such that

$$
d\left(y, S_{P}\left(x_{0}\right)\right) \leq \alpha d\left(0, \mathbf{F}_{P}^{\text {nat }}\left(x_{0}, y\right)\right)=\alpha\left\|\mathbf{F}_{P}^{\text {nat }}\left(x_{0}, y\right)\right\|
$$

for all $y \in S_{Q}(x) \cap B_{m}\left(y^{*}, \delta_{1}\right)$ with $x \in B_{n}\left(x_{0}, \delta_{2}\right) \cap \mathcal{X}$ and $Q \in U\left(P, \delta_{3}\right)$. Let $y \in S_{Q}(x)$. Then $\mathbf{F}_{Q}^{\text {nat }}(x, y)=0$ and

$$
\left\|\mathbf{F}_{P}^{\text {nat }}\left(x_{0}, y\right)\right\|=\left\|\mathbf{F}_{Q}^{\text {nat }}(x, y)-\mathbf{F}_{P}^{\text {nat }}\left(x_{0}, y\right)\right\|
$$

Combining (4.20) and (4.21), and making use of Proposition 4.1, we obtain

$$
d\left(y, S_{P}\left(x_{0}\right)\right) \leq \alpha\left\|\mathbf{F}_{Q}^{\mathrm{nat}}(x, y)-\mathbf{F}_{P}^{\mathrm{nat}}\left(x_{0}, y\right)\right\| \leq \alpha \rho\left(\mathscr{D}_{H}(Q, P)+\left\|x-x_{0}\right\|\right)^{\beta}
$$

for any $y \in S_{Q}(x) \cap B_{m}\left(y^{*}, \delta_{1}\right)$ with $Q \in U\left(P, \delta_{2}^{\prime}\right)$ and $x \in B_{n}\left(x_{0}, \delta_{3}^{\prime}\right) \cap \mathcal{X}$, where $\delta_{2}^{\prime}=\min \left\{\delta_{2}, \bar{\delta}\right\}$ and $\delta_{3}^{\prime}=\min \left\{\delta_{3}, \bar{\delta}\right\}$. Analogous to the second part of the proof of Lemma 2.2, we can show by exploiting the compactness of $S_{P}\left(x_{0}\right)$ that there exists $\varepsilon>0$ such that

$$
d\left(y, S_{P}\left(x_{0}\right)\right) \leq \alpha \rho\left(\mathscr{D}_{H}(Q, P)+\left\|x-x_{0}\right\|\right)^{\beta}
$$

for every $y \in S_{Q}(x) \cap\left(S_{P}\left(x_{0}\right)+\varepsilon \mathcal{B}_{m}\right)$. The conclusion is apparent in that we have shown in Part (i) $S_{Q}(x) \subseteq S_{P}\left(x_{0}\right)+\varepsilon \mathcal{B}_{m}$.

Part (iii). The locally Lipschitz homeomorphism of $\mathbf{F}_{P}^{\text {nat }}\left(x_{0}, y\right)$ at $y^{*}$ implies that there exist positive constants $\delta, \varepsilon$ and a unique locally Lipschitz continuous function $\tilde{y}(\cdot): B_{m}(0, \delta) \rightarrow B_{m}\left(y^{*}, \varepsilon\right)$ such that

$$
\tilde{y}(0)=y^{*},\left\{y^{*}\right\}=S_{P}\left(x_{0}\right) \cap B_{m}\left(y^{*}, \varepsilon\right) \quad \text { and } \quad \mathbf{F}_{P}^{\text {nat }}\left(x_{0}, \cdot\right)^{-1}(q) \cap B_{m}\left(y^{*}, \varepsilon\right)=\tilde{y}(q)
$$

for every $q \in B_{m}(0, \delta)$. For each $Q$ and $x$, let

$$
\Delta_{Q}(x, y):=\mathbf{F}_{Q}^{\text {nat }}(x, y)-\mathbf{F}_{P}^{\text {nat }}\left(x_{0}, y\right)
$$

It is easy to see that $\Delta_{Q}(x, y)$ is a continuous vector-valued function of $y$ parameterized by $Q$ and $x$. By Proposition 4.1, there exists positive number $\delta_{1}$ such that

$$
\sup _{x \in B_{n}\left(x_{0}, \delta_{1}\right), Q \in U\left(P, \delta_{1}\right), y \in B_{m}\left(y^{*}, \varepsilon\right) \cap \mathcal{Y}}\left\|\Delta_{Q}(x, y)\right\|<\delta,
$$


which implies that $\Delta_{Q}(x, y) \in B_{m}(0, \delta)$. Thus for each $\hat{y} \in S_{Q}(x) \cap B_{m}\left(y^{*}, \varepsilon\right)$ with $x \in B_{n}\left(x_{0}, \delta_{1}\right)$ and $Q \in U\left(P, \hat{\delta}_{1}\right), \hat{y}$ satisfies $\mathbf{F}_{P}^{\text {nat }}(x, \hat{y})=\Delta_{Q}(x, \hat{y})$, or equivalently, $\mathbf{F}_{P}^{\text {nat }}(x, \cdot)^{-1}\left(\Delta_{Q}(x, \hat{y})\right)=\hat{y}$. By the definition of $\tilde{y}(\cdot)$ and Proposition 4.1, we have $\tilde{y}\left(\Delta_{Q}(x, \hat{y})\right)=\hat{y}$ and there exists $K>0$ and $\mu>0$ such that

$$
\begin{aligned}
\left\|\hat{y}-y^{*}\right\| & =\left\|\tilde{y}\left(\Delta_{Q}(x, \hat{y})\right)-\tilde{y}(0)\right\| \leq K\left\|\Delta_{Q}(x, y)\right\| \\
& \leq K \sup _{x \in B_{n}\left(x_{0}, \delta_{1}\right), Q \in U\left(P, \delta_{1}\right), y \in B_{m}\left(y^{*}, \varepsilon\right) \cap \mathcal{Y}}\left\|\Delta_{Q}(x, y)\right\| \\
& \leq K \mu\left(\mathscr{D}_{H}(Q, P)+\left\|x-x_{0}\right\|\right)^{\beta} .
\end{aligned}
$$

The proof is complete.

To help readers better understand the SQVIP model (1.1) and the stability results Theorem 4.1, we present an example before we conclude this section.

Example 4.1 Let us revisit Example 3.1. Let $P_{N}$ be an empirical measures over set $\xi^{1}, \cdots, \xi^{N}$, i.e.,

$$
P_{N}(\cdot):=\frac{1}{N} \sum_{k=1}^{N} \mathbb{1}_{\xi^{k}}(\cdot)
$$

where $\xi^{1}, \cdots, \xi^{N}$ are independent and identically distributed random variables having the same distribution as $\xi$ and $\mathbb{1}_{\xi^{k}}$ is defined by

$$
\mathbb{1}_{\xi^{k}}(\xi(\omega))= \begin{cases}1, & \text { if } \xi(\omega)=\xi^{k} \\ 0, & \text { if } \xi(\omega) \neq \xi^{k}\end{cases}
$$

In this case, $\mathbb{E}_{P_{N}}[\xi]=\frac{1}{N} \sum_{k=1}^{N} \xi^{k}$. By the well known Glivenko-Cantelli theorem [38], $P_{N}$ converges to $P$ weakly as $N$ increases and under the pseudo metric with $\mathcal{G}$ being generated by $e^{y \xi}$ for $y \in[-1,1]$ as discussed in Section 2.4. The resulting perturbed problem of (3.9) is

$$
0 \in x^{2}+\frac{1}{N} \sum_{k=1}^{N} e^{y \xi^{k}}+\mathcal{N}_{\widehat{\Gamma}_{N}(x, y)}(y), x, y \in \mathbb{R}
$$

with

$$
\widehat{\Gamma}_{N}(x, y):=\left\{z \in[-1,1]:\left(x^{2}-\frac{1}{N} \sum_{k=1}^{N} e^{y \xi^{k}} y\right) z \geq 0\right\} .
$$

Analogous to the analysis in Example 3.1, we have for $(x, y) \in[-e-0.5, e+0.5] \times[-1,1]$,

$$
\mathcal{N}_{\widehat{\Gamma}_{N}(x, y)}(y)=\left\{\begin{aligned}
\mathbb{R}_{-}, & \text {for } x^{2}>\frac{1}{N} \sum_{k=1}^{N} e^{y \xi^{k}} y, y=0, \\
\{0\}, & \text { for } x^{2}>\frac{1}{N} \sum_{k=1}^{N} e^{y \xi^{k}} y, y \in(0,1) \text { or } x^{2}=\frac{1}{N} \sum_{k=1}^{N} e^{y \xi^{k}} y, y \in[0,1), \\
\mathbb{R}_{+}, & \text {for } x^{2}>\frac{1}{N} \sum_{k=1}^{N} e^{y \xi^{k}} y, y=1 \text { or } x^{2}=\frac{1}{N} \sum_{k=1}^{N} e^{y \xi^{k}} y, y=1, \\
\emptyset, & \text { otherwise. }
\end{aligned}\right.
$$


Let $\tilde{S}_{N}(x)$ denote the set of solutions to (4.23). Then

$$
\tilde{S}_{N}(x)=\left\{y \in[-1,1]: 0 \in x^{2}+\frac{1}{N} \sum_{k=1}^{N} e^{y \xi^{k}}+\mathcal{N}_{\Gamma_{P}(x, y)}(y)\right\}=\{0\}
$$

for $x \in[-e-0.5, e+0.5] \backslash\{0\}$. Recall that in Example 3.1 we have shown that $\tilde{S}\left(x_{0}\right)=\emptyset$. Thus $\mathbb{D}\left(\tilde{S}_{N}(x), \tilde{S}\left(x_{0}\right)\right) \nrightarrow 0$ as $x$ converges to $x_{0}$ and $N$ tends to infinity.

Let $x_{0}=e$, where $e$ is the Euler number. For any $y \in[-1,1]$,

$$
0 \in \operatorname{int}\left\{\left(x_{0}^{2}-\mathbb{E}_{P}\left[e^{y \xi}\right] y\right)[-1,1]-\mathbb{R}_{+}\right\}
$$

due to the fact that $\mathbb{E}_{P}\left[e^{y \xi}\right] y \in[-e, e]$ and $x_{0}^{2}-\mathbb{E}_{P}\left[e^{y \xi}\right] y>0$. Hence the SCQ (3.1) in Theorem 4.1 holds. Then by Theorem 4.1, there exist $\rho>0$ and $\beta \in(0,1)$ such that

$$
\mathbb{D}\left(\tilde{S}_{N}(x), \tilde{S}\left(x_{0}\right)\right) \leq \Upsilon\left(2 \rho\left(A_{N}+\left\|x-x_{0}\right\|\right)^{\beta}\right)
$$

when $x$ is in a neighborhood of $x_{0}$ and $N$ is sufficiently large, where

$$
A_{N}=\sup _{(y, z) \in[-1,1] \times[-1,1]}\left|(z+1)\left(\mathbb{E}_{P}\left[e^{y \xi}\right]-\frac{1}{N} \sum_{k=1}^{N} e^{y \xi^{k}}\right)\right|
$$

and for $\varepsilon \in(0,1)$,

$$
\begin{aligned}
R(\varepsilon) & =\inf _{y \in[-1,1], d\left(y, \tilde{S}\left(x_{0}\right)\right) \geq \varepsilon}\left|\Pi_{\Gamma_{P}\left(x_{0}, y\right)}\left(y-\mathbb{E}_{P}\left[F\left(x_{0}, y, \xi\right)\right]\right)-y\right| \\
& =\inf _{y \in[-1,1],|y| \geq \varepsilon}\left|\Pi_{[0,1]}\left(y-x_{0}^{2}-\mathbb{E}_{P}\left[e^{y \xi}\right]\right)-y\right| \\
& =\inf _{y \in[-1,1],|y| \geq \varepsilon}|-y|=\varepsilon
\end{aligned}
$$

due to the fact that $y-x_{0}^{2}-\mathbb{E}_{P}\left[e^{y \xi}\right]<0$ and $\tilde{S}\left(x_{0}\right)=\{0\}$, where $\tilde{S}\left(x_{0}\right)=\{0\}$ is directly from

$$
\mathcal{N}_{\Gamma_{P}\left(x_{0}, y\right)}(y)=\mathcal{N}_{[0,1]}(y)=\left\{\begin{array}{cl}
\mathbb{R}_{-}, & \text {for } y=0, \\
\{0\}, & \text { for } y \in(0,1), \\
\mathbb{R}_{+}, & \text {for } y=1, \\
\emptyset, & \text { otherwise }
\end{array}\right.
$$

according to (3.10) and $x_{0}^{2}=e^{2}>\mathbb{E}_{P}\left[e^{y \xi}\right] y, y \in[-1,1]$. Since $A_{N} \rightarrow 0$ w.p.1 as $N \rightarrow \infty$, we obtain $\mathbb{D}\left(\tilde{S}_{N}(x), \tilde{S}\left(x_{0}\right)\right) \rightarrow 0$ w.p.1 as $N \rightarrow \infty$ and $x \rightarrow x_{0}$ directly from $(5.34)$ and $R^{-1}(\varepsilon)=\varepsilon \rightarrow 0$ as $\varepsilon \downarrow 0$. Indeed, by (4.23), $\tilde{S}_{N}(x)=\{0\}$ for $x \in(e-0.5, e+0.5)$ due to the fact that $\mathcal{N}_{\widehat{\Gamma}_{N}(x, y)}(y)=\mathcal{N}_{[0,1]}(y)$ for $y \in[-1,1]$. Therefore we have $\mathbb{D}\left(\tilde{S}_{N}(x), \tilde{S}\left(x_{0}\right)\right) \rightarrow 0$ as $x$ converges to $x_{0}$ and $N$ tends to infinity.

\section{Applications}

In this section, we discuss application of the stability results that we have established in the preceding section for the SQVIP (1.1). Since the SQVIP model can be used to represent the first order optimality conditions of many one stage stochastic optimization or equilibrium problems with stochastic cone constraints, we restrict our discussion to the problems where there is a clear advantage to use our newly established results over other existing results on SVIP in the literature. 


\subsection{One stage stochastic programs with stochastic semidefinite constraints}

Let us start with a mathematical program with matrix cone constraints:

$$
\begin{array}{cl}
\min _{y \in \mathbb{R}^{m}} & \mathbb{E}_{P}[v(x, y, \xi)] \\
\text { s.t. } & \left(\mathbb{E}_{P}[g(x, y, \xi)]-\mu_{0}\right)^{T} \Sigma_{0}^{-1}\left(\mathbb{E}_{P}[g(x, y, \xi)]-\mu_{0}\right) \leq \gamma, \\
& \mathbb{E}_{P}\left[\left(g(x, y, \xi)-\mu_{0}\right)\left(g(x, y, \xi)-\mu_{0}\right)^{T}\right] \preceq \Sigma_{0}, \\
& y \in \mathcal{Y},
\end{array}
$$

where $v, g$ are continuous functions mapping from $\mathbb{R}^{n} \times \mathbb{R}^{m} \times \Xi$ to $\mathbb{R}$ and $\mathbb{R}^{p}$ respectively, $x \in \mathbb{R}^{n}$ is a parameter, $\xi: \Omega \rightarrow \Xi$ be a random vector with $k$ components defined on a probability space $(\Omega, \mathcal{F}, P), \mathbb{E}_{P}[\cdot]$ denotes the mathematical expectation with respect to the probability measure $P$ and $\mathcal{Y}$ is a closed convex subset of $\mathbb{R}^{m}, \mu_{0}$ is a fixed vector and $\Sigma_{0}$ is a symmetric positive definite matrix. Here and later, for a matrix $M$, we write $M \preceq 0$ and $M \succeq 0$ for $M$ being negative semidefinite and positive semidefinite and $M \prec 0$ and $M \succ 0$ for $M$ being negative definite and positive definite.

In the context of portfolio optimization, we may interpret $g(x, y, \xi)$ as a vector of returns from several portfolios, $\mu_{0}$ as the targeted mean value and $\Sigma_{0}$ a specified level of covariance. The model differs from existing models in the literature of portfolio optimization where $g$ is often a real valued function representing aggregate return from a single portfolio of investments. By considering $g$ as a vector valued function, the model allows one to divide the portfolio into a number of groups according to the nature of the assets and the constraint on covariance restricts correlation between the groups of the assets. Through a simple mathematical maneuver, we can reformulate (5.25) as

$$
\begin{array}{ll}
\min _{y \in \mathbb{R}^{m}} & \mathbb{E}_{P}[v(x, y, \xi)] \\
\text { s.t. } & \mathbb{E}_{P}\left[\begin{array}{ll}
\Sigma_{0} & g(x, y, \xi)-\mu_{0} \\
\left(g(x, y, \xi)-\mu_{0}\right)^{T} & \gamma
\end{array}\right] \succeq 0, \\
& \mathbb{E}_{P}\left[\left(g(x, y, \xi)-\mu_{0}\right)\left(g(x, y, \xi)-\mu_{0}\right)^{T}\right] \preceq \Sigma_{0}, \\
& y \in \mathcal{Y} .
\end{array}
$$

Let $\mathcal{S}^{p}$ and $\mathcal{S}_{+}^{p}$ denote the space of $p \times p$ symmetric matrices and the cone of positive semidefinite matrices in $\mathcal{S}^{p}$ respectively. Problem (5.26) can be recast as a one stage stochastic semidefinite programming (SSDP):

$$
\begin{array}{cl}
\min _{y \in \mathbb{R}^{m}} & \mathbb{E}_{P}[v(x, y, \xi)] \\
\text { s.t. } & \mathbb{E}_{P}[G(x, y, \xi)] \in \mathcal{K}, \\
& y \in \mathcal{Y}
\end{array}
$$

where

$$
G(x, y, \xi)=\left[\begin{array}{cc}
\Sigma_{0} & g(x, y, \xi)-\mu_{0} \\
\left(g(x, y, \xi)-\mu_{0}\right)^{T} & \gamma
\end{array}\right] \otimes\left[\Sigma_{0}-\left(g(x, y, \xi)-\mu_{0}\right)\left(g(x, y, \xi)-\mu_{0}\right)^{T}\right],
$$

and $\mathcal{K}=\mathcal{S}_{+}^{2 p} \otimes \mathcal{S}_{+}^{p}$, where " $\otimes$ " denotes the Cartesian product. In the case when $g(x, \cdot, \xi)$ is linear function in $y$ for any fixed $x \in \mathcal{X}$ and $\xi \in \Xi$, we can show that

$$
\Gamma_{P}(x):=\left\{z \in \mathcal{Y}: \mathbb{E}_{P}[G(x, z, \xi)] \in \mathcal{K}\right\}
$$


is a convex set for any fixed $x \in \mathcal{X}$. Consequently, the solution set of SSDP can be represented as the solution set of $\operatorname{SQVIP}(1.1)$ with $\Gamma_{P}(x, y):=\Gamma_{P}(x)$ being defined in (5.28) and $F(x, y, \xi):=$ $\nabla_{y} v(x, y, \xi)$.

Evidently, if the integral involved in the mathematical expectation can be evaluated either analytically or numerically, then the SSDP can be solved by existing numerical methods. However, in many situations, exact evaluation of the expected value is either impossible or prohibitively expensive. Consequently, some approximation methods such as SAA are needed to deal with the mathematical expectation and it is necessary to assess the impact on the optimal value and optimization solution. Here we take a general step by looking into stability of the problem in terms of variation of probability measures which should cover abroad class of approximation schemes with practical interest. Specifically, we consider

$$
\begin{array}{cl}
\min _{y \in \mathbb{R}^{m}} & \mathbb{E}_{Q}[v(x, y, \xi)] \\
\text { s.t. } & \mathbb{E}_{Q}[G(x, y, \xi)] \in \mathcal{K}, \\
& y \in \mathcal{Y}
\end{array}
$$

where probability measure $Q$ is an approximation of $P$ under some appropriate metric.

Assumption 5.1 Let $\hat{\mathcal{P}} \subset \mathscr{P}$ be a set of probability measures such that $P, Q \in \hat{\mathcal{P}}$. The following hold.

(a) For each fixed $\xi \in \Xi$ and $x \in \mathcal{X}, \nabla_{y} v(x, y, \xi)$ is uniformly Lipschitz continuous in $y$ with Lipschitz modulus being bounded by $\kappa(\xi)$, where $\sup _{P \in \hat{\mathcal{P}}} \mathbb{E}_{P}[\kappa(\xi)]<\infty$.

(b) For each fixed $\xi \in \Xi$ and $x \in \mathcal{X}, \nabla_{y} v(x, y, \xi)$ is $\theta$-monotone on $\mathbb{R}^{m}$ for some $\theta>1$, i.e., there exists $c(\xi)>0$ such that

$$
\left(\nabla_{y} v(x, y, \xi)-\nabla_{y} v\left(x, y^{\prime}, \xi\right)\right)^{T}\left(y-y^{\prime}\right) \geq c(\xi)\left\|y-y^{\prime}\right\|^{\theta}
$$

holds for any $y, y^{\prime} \in \mathcal{Y}$ and each fixed $x \in \mathcal{X}$ and $\xi \in \Xi$ with $\sup _{P \in \hat{\mathcal{P}}} \mathbb{E}_{P}[c(\xi)]<\infty$. Unless specified otherwise, $\|\cdot\|$ denotes the Euclidean norm in a finite dimensional space throughout this section.

Theorem 5.1 Let $\widehat{S}_{P}(x)$ and $\widehat{S}_{Q}(x)$ denote the set of solutions to problems (5.27) and (5.29) respectively. Let $\mathcal{X} \subseteq \mathbb{R}^{n}$ and $\mathcal{Y} \subseteq \mathbb{R}^{m}$ be compact sets. Assume: (a) $g(x, \cdot, \xi)$ be a linear function in $y$ for any fixed $x \in \mathcal{X}$ and $\xi \in \Xi$, (b) Assumption 3.2 holds for $F(x, y, \xi) \equiv \nabla_{y} v(x, y, \xi)$ and $G(x, y, \xi)$ defined in (5.27), (c) for a fixed $x_{0} \in \mathcal{X}$, there exists $\hat{y} \in \mathcal{Y}$ such that

$$
\mathbb{E}_{P}\left[\begin{array}{cc}
\Sigma_{0} & g\left(x_{0}, \hat{y}, \xi\right)-\mu_{0} \\
\left(g\left(x_{0}, \hat{y}, \xi\right)-\mu_{0}\right)^{T} & \gamma
\end{array}\right] \succ 0 \text { and } \mathbb{E}_{P}\left[\left(g\left(x_{0}, \hat{y}, \xi\right)-\mu_{0}\right)\left(g\left(x_{0}, \hat{y}, \xi\right)-\mu_{0}\right)^{T}\right] \prec \Sigma_{0} .
$$

Then the following assertions hold.

(i) There exist $\delta>0, \rho>0$ and $\beta \in(0,1)$ such that

$$
\mathbb{D}\left(\widehat{S}_{Q}(x), \widehat{S}_{P}\left(x_{0}\right)\right) \leq \Upsilon\left(2 \rho\left(\mathscr{D}_{H}(Q, P)+\left\|x-x_{0}\right\|\right)^{\beta}\right)
$$


for any $(x, Q) \in B_{n}\left(x_{0}, \delta\right) \cap \mathcal{X} \times U(P, \delta)$, where for any small positive number $\varepsilon>0$,

$$
R(\varepsilon)=\inf _{y \in \mathcal{Y}, d\left(y, \widehat{S}_{P}\left(x_{0}\right)\right) \geq \varepsilon}\left\|\Pi_{\Gamma_{P}\left(x_{0}\right)}\left(y-\mathbb{E}_{P}\left[\nabla_{y} v\left(x_{0}, y, \xi\right)\right]\right)-y\right\|
$$

$\Upsilon(t)=\min \left\{\varepsilon \in \mathbb{R}_{+}: R(\varepsilon)=t\right\}$ and $\Upsilon(t) \rightarrow 0$ as $t \downarrow 0$.

(ii) If, in addition, $\nabla_{y} v(x, y, \xi)$ satisfies Assumption 5.1, then $\widehat{S}_{Q}(x)$ and $\widehat{S}_{P}\left(x_{0}\right)$ reduce to singleton and there exist positive constants $\alpha, \rho$ and $\beta$ such that

$$
\mathbb{D}\left(\widehat{S}_{Q}(x), \widehat{S}_{P}\left(x_{0}\right)\right) \leq \rho\left(\mathscr{D}_{H}(Q, P)+\left\|x-x_{0}\right\|\right)^{\alpha}
$$

for any $(x, Q) \in B_{n}\left(x_{0}, \delta\right) \cap \mathcal{X} \times U(P, \delta)$,

where $\mathscr{D}_{H}(Q, P)$ is defined as in Section 2.3 with $F=\nabla_{y} v$ and $G$ being defined as in this theorem.

Proof. The thrust of the proof is to apply Theorem 4.1. Let $W$ in Theorem 4.1 be defined as $\mathcal{S}^{2 p} \otimes \mathcal{S}^{p}$ equipped with Frobenius norm.

Recall that $\mathcal{K}=\mathcal{S}_{+}^{2 p} \otimes \mathcal{S}_{+}^{p}$. It is easy to see that int $\mathcal{K}$ is nonempty and $\mathbb{E}_{P}\left[G\left(x_{0}, \cdot, \xi\right)\right]$ is $-\mathcal{K}$-convex. Moreover, condition (c) is equivalent to $\mathbb{E}_{P}\left[G\left(x_{0}, \hat{y}, \xi\right)\right] \in \operatorname{int} \mathcal{S}_{+}^{2 p} \otimes \mathcal{S}_{+}^{p}$, and by [5, Proposition 2.106], it coincides with the Slater condition (3.1). The conclusion follows from Theorem 4.1 (i).

Part (ii). Assumption 5.1 (b) implies that $\mathbb{E}_{P}\left[\nabla_{y} v\left(x_{0}, y, \xi\right)\right]$ is $\theta$-monotone over $\mathcal{Y}$. By [11, Theorem 2.3.3], $\widehat{S}_{P}\left(x_{0}\right)$ is a singleton, denoted by $\left\{y^{*}\right\}$. Moreover, there exists a constant $\kappa>0$ such that

$$
\left\|y-y^{*}\right\| \leq \kappa\left\|\Pi_{\Gamma_{P}\left(x_{0}\right)}\left(y-\mathbb{E}_{P}\left[\nabla_{y} v\left(x_{0}, y, \xi\right)\right]\right)-y\right\|^{\frac{1}{\theta-1}}, \forall y \in \mathcal{Y} .
$$

Thus for any $y \in \widehat{S}_{Q}(x)$,

$$
\left\|y-y^{*}\right\| \leq \kappa\left\|\Pi_{\Gamma_{Q}(x)}\left(y-\mathbb{E}_{Q}\left[\nabla_{y} v(x, y, \xi)\right]\right)-\Pi_{\Gamma_{P}\left(x_{0}\right)}\left(y-\mathbb{E}_{P}\left[\nabla_{y} v\left(x_{0}, y, \xi\right)\right]\right)\right\|^{\frac{1}{\theta-1}} .
$$

Furthermore, under Assumptions 3.2 and 3.2, it follows by Proposition 4.1 that there exist positive constants $L$ and $\beta \in(0,1)$ such that

$$
\left\|\Pi_{\Gamma_{Q}(x)}\left(y-\mathbb{E}_{Q}\left[\nabla_{y} v(x, y, \xi)\right]\right)-\Pi_{\Gamma_{P}\left(x_{0}\right)}\left(y-\mathbb{E}_{P}\left[\nabla_{y} v\left(x_{0}, y, \xi\right)\right]\right)\right\| \leq L\left(\mathscr{D}_{H}(Q, P)+\left\|x-x_{0}\right\|\right)^{\beta} .
$$

The conclusion follows.

Remark 5.1 A sufficient condition for $\mathbb{E}_{P}\left[\left(g\left(x_{0}, \hat{y}, \xi\right)-\mu_{0}\right)\left(g\left(x_{0}, \hat{y}, \xi\right)-\mu_{0}\right)^{T}\right] \prec \Sigma_{0}$ is the smallest eigenvalue of $\Sigma_{0}$ being greater than $\left\|\mathbb{E}_{P}\left[g\left(x_{0}, \hat{y}, \xi\right)\right]-\mu_{0}\right\|^{2}$.

To help readers better understand the theory developed in this section, we consider a simple example. 
Example 5.1 Suppose that we have a fund normalized to 1 to be invested in three areas: A, B and C. Area A involves 2 assets whereas $\mathrm{B}$ and $\mathrm{C}$ each involves only one asset. The rate of return from each asset is stochastic, denoted by $\xi_{i}, i=1, \cdots, 4$. Let $y_{i}$ denote the proportion of the fund to be invested in asset $i$. The return from each area can be written respectively as $y_{1} \xi_{1}+y_{2} \xi_{2}, y_{2} \xi_{3}$ and $y_{4} \xi_{4}$. Let $x_{1}, x_{2}, x_{3}$ denote some kind of "weight" on the return of the three investment areas (we don't require $\sum_{i=1}^{3}=1$ ). The total weighted portfolio return is

$$
v(x, y, \xi)=x_{1}\left(y_{1} \xi_{1}+y_{2} \xi_{2}\right)+x_{2} y_{3} \xi_{3}+x_{3} y_{4} \xi_{4} .
$$

Let $g_{1}(x, y, \xi)=x_{1}\left(y_{1} \xi_{1}+y_{2} \xi_{2}\right), g_{2}(x, y, \xi)=x_{2} y_{3} \xi_{3}$ and $g_{3}(x, y, \xi)=x_{3} y_{4} \xi_{4}$, and write $g$ for $\left(g_{1}, g_{2}, g_{3}\right)$. We require the mean and covariance of $g_{i}$ within a specified range, that is,

$$
\begin{aligned}
\left(\mathbb{E}_{P}[g(x, y, \xi)]-(1,1,0.5)^{T}\right)^{T}\left(\mathbb{E}_{P}[g(x, y, \xi)]-(1,1,0.5)^{T}\right) & \leq 0.5, \\
\mathbb{E}_{P}\left[\left(g(x, y, \xi)-(1,1,0.5)^{T}\right)\left(g(x, y, \xi)-(1,1,0.5)^{T}\right)^{T}\right] & \preceq I,
\end{aligned}
$$

where $I$ is a 3 by 3 identity matrix and $P$ is a joint probability distribution of $\xi=\left(\xi_{1}, \xi_{2}, \xi_{3}, \xi_{4}\right)$. Assume $\xi_{2}$ and $\xi_{3}$ are correlated with the following joint distribution $\xi_{1}$ and $\xi_{4}$ are independent with uniform

\begin{tabular}{c|cc|c}
$\xi_{2} \backslash \xi_{3}$ & 3 & 2 & Marginal prob \\
\hline 3 & 0.1 & 0.5 & 0.6 \\
2 & 0.3 & 0.1 & 0.4 \\
\hline Marginal prob & 0.4 & 0.6 &
\end{tabular}

Table 1: Joint probability distribution

distribution over $[-1,1]$ and $[-2,7]$. Let $x_{0}=(1,1,2)$ and $\mathcal{Y}=\left\{\left(y_{1}, y_{2}, y_{3}, y_{4}\right) \in \mathbb{R}^{4}: y_{1}+y_{2}+y_{3}+y_{4}=\right.$ $\left.1,0 \leq y_{i} \leq 1, i=1,2,3,4.\right\}$. We show condition (5.30) holds. By simple computing, we have $\mathbb{E}_{P}\left[\xi_{1}\right]=0, \mathbb{E}_{P}\left[\xi_{2}\right]=2.6, \mathbb{E}_{P}\left[\xi_{3}\right]=2.4, \mathbb{E}_{P}\left[\xi_{4}\right]=2.5, \mathbb{E}_{P}\left[\xi_{1}^{2}\right]=\frac{1}{3}, \mathbb{E}_{P}\left[\xi_{2}^{2}\right]=7, \mathbb{E}_{P}\left[\xi_{3}^{2}\right]=6, \mathbb{E}_{P}\left[\xi_{4}^{2}\right]=13$. Let $\bar{y}=(0.2,0.5,0.2,0.1)$, we have

$$
\begin{aligned}
& \left(\mathbb{E}_{P}\left[g\left(x_{0}, \bar{y}, \xi\right)\right]-(1,1,0.5)^{T}\right)^{T}\left(\mathbb{E}_{P}\left[g\left(x_{0}, \bar{y}, \xi\right)\right]-(1,1,0.5)^{T}\right) \\
= & (0.3)^{2}+(-0.52)^{2}+0^{2}=0.3604<0.5 .
\end{aligned}
$$

For any $h \in \mathbb{R}^{3} \backslash\{0\}$,

$$
\begin{aligned}
& h^{T}\left(\mathbb{E}_{P}\left[\left(g\left(x_{0}, \bar{y}, \xi\right)-(1,1,0.5)^{T}\right)\left(g\left(x_{0}, \bar{y}, \xi\right)-(1,1,0.5)^{T}\right)^{T}\right]-I\right) h \\
= & \left.\mathbb{E}_{P}\left[\left(g\left(x_{0}, \bar{y}, \xi\right)-(1,1,0.5)^{T}\right)^{T} h\right)^{2}\right]-\|h\|^{2} \\
\leq & \left(\mathbb{E}_{P}\left[\left\|g\left(x_{0}, \bar{y}, \xi\right)-(1,1,0.5)^{T}\right\|^{2}\right]-1\right)\|h\|^{2}<0
\end{aligned}
$$

due to the fact that

$$
\mathbb{E}_{P}\left[\left\|g\left(x_{0}, \bar{y}, \xi\right)-(1,1,0.5)^{T}\right\|^{2}\right]=0.7+\frac{0.04}{3}<1 .
$$

Therefore the inequalities (5.32) and (5.33) imply the Slater condition (5.30) in Theorem 5.1. By Theorem 5.1, there exist constants $\rho>0, \delta>0$ and $\beta \in(0,1)$ such that

$$
\mathbb{D}\left(\widehat{S}_{Q}(x), \widehat{S}_{P}\left(x_{0}\right)\right) \leq \Upsilon\left(\rho\left(\mathscr{D}_{H}(Q, P)+\left\|x-x_{0}\right\|\right)^{\beta}\right)
$$

for any $(x, Q) \in B_{n}\left(x_{0}, \delta\right) \times U(P, \delta)$, which means that

$$
\mathbb{D}\left(\widehat{S}_{Q}(x), \widehat{S}_{P}\left(x_{0}\right)\right) \rightarrow 0
$$


as $x$ converges to $x_{0}$ and $Q$ converges to $P$ under pseudometric $\mathscr{D}_{H}$ defined in Section 2.4.

We solve problem (5.25) via a Matlab solver PENLB in [10] and obtain an approximate optimal solution $y_{0}=(0.5074,0.2276,0.2466,0.0184)^{T}$. To investigate the impact of the variation of $x$ and $P$ on the optimal solution, we have carried out some numerical experiments through PENLB installed in Matlab 2010a running on a PC with Intel Pentium M of $1.60 \mathrm{GHz}$ CPU with different values of parameter $x$ and probability measure $P$. In the tables below, we use $y(x, Q)$ and $\left\|y(x, Q)-y_{0}\right\|$ to denote the optimal solution of problem (5.25) with parameter $x$ and probability measure $Q$ and the error of optimal solutions respectively. In the experiments, variation of the probability measure is reflected by the change of samples. We use the random number generator unifrnd and rand in the Matlab to generate iid samples $\left\{\xi^{1}, \xi^{2}, \cdots, \xi^{N}\right\}$. The test results are summarized in the following three tables.

Table 2: Stability analysis with variation of $x$.

\begin{tabular}{|c|c|c|}
\hline$x$ & $y(x, P)$ & ||$y(x, P)-y_{0} \mid$ \\
\hline$(0.70,0.70,1.70)$ & $\left(\begin{array}{llll}0.3011 & 0.3251 & 0.3522 & 0.0216\end{array}\right)$ & 0.2515 \\
\hline$(0.80,0.80,1.80)$ & $\left(\begin{array}{llll}0.3869 & 0.2845 & 0.3082 & 0.0204\end{array}\right)$ & 0.1468 \\
\hline$(0.85,0.85,1.85)$ & $\left(\begin{array}{llll}0.4223 & 0.2678 & 0.2901 & 0.0198\end{array}\right)$ & 0.1037 \\
\hline$(0.90,0.90,1.90)$ & $\left(\begin{array}{llll}0.4538 & 0.2529 & 0.2740 & 0.0193\end{array}\right)$ & 0.0653 \\
\hline$(0.95,0.95,1.95)$ & $\left(\begin{array}{llll}0.4821 & 0.2396 & 0.2595 & 0.0188\end{array}\right)$ & 0.0308 \\
\hline$(0.99,0.99,1.99)$ & $\left(\begin{array}{llll}0.5026 & 0.2299 & 0.2491 & 0.0184\end{array}\right)$ & 0.0059 \\
\hline
\end{tabular}

Table 3: Stability analysis with variation of sample size $N$.

\begin{tabular}{|c|c|c|}
\hline$N$ & $y\left(x_{0}, P_{N}\right)$ & $\left|y\left(x_{0}, P_{N}\right)-y_{0}\right| \mid$ \\
\hline 100 & $\left(\begin{array}{llll}0.5264 & 0.2113 & 0.2435 & 0.0187\end{array}\right)$ & 0.0252 \\
\hline 200 & $\left(\begin{array}{llll}0.5104 & 0.2238 & 0.2486 & 0.0172\end{array}\right)$ & 0.0054 \\
\hline 500 & $\left(\begin{array}{llll}0.5096 & 0.2241 & 0.2470 & 0.0194\end{array}\right)$ & 0.0043 \\
\hline 1000 & $\left(\begin{array}{llll}0.5069 & 0.2261 & 0.2475 & 0.0195\end{array}\right)$ & 0.0021 \\
\hline 2000 & $\left(\begin{array}{llll}0.5063 & 0.2273 & 0.2474 & 0.0190\end{array}\right)$ & 0.0015 \\
\hline 4000 & $\left(\begin{array}{llll}0.5078 & 0.2265 & 0.2471 & 0.0186\end{array}\right)$ & 0.0013 \\
\hline
\end{tabular}

Table 4: Stability analysis with variation of both $x$ and $N$.

\begin{tabular}{|c|c|c|c|}
\hline$x$ & $N$ & $y\left(x, P_{N}\right)$ & $\left\|y\left(x, P_{N}\right)-y_{0}\right\|$ \\
\hline$(0.70,0.70,1.70)$ & 100 & $\left(\begin{array}{llll}0.3183 & 0.3118 & 0.3479 & 0.0221\end{array}\right)$ & 0.2304 \\
\hline$(0.80,0.80,1.80)$ & 200 & $\left(\begin{array}{llll}0.3900 & 0.2801 & 0.3108 & 0.0191\end{array}\right)$ & 0.1438 \\
\hline$(0.85,0.85,1.85)$ & 500 & $\left(\begin{array}{llll}0.4249 & 0.2636 & 0.2906 & 0.0210\end{array}\right)$ & 0.1002 \\
\hline$(0.90,0.90,1.90)$ & 1000 & $\left(\begin{array}{llll}0.4526 & 0.2519 & 0.2750 & 0.0205\end{array}\right)$ & 0.0663 \\
\hline$(0.95,0.95,1.95)$ & 2000 & $\left(\begin{array}{llll}0.4809 & 0.2392 & 0.2604 & 0.0195\end{array}\right)$ & 0.0321 \\
\hline$(0.99,0.99,1.99)$ & 4000 & $\left(\begin{array}{llll}0.5029 & 0.2288 & 0.2496 & 0.0187\end{array}\right)$ & 0.0056 \\
\hline
\end{tabular}

The numerical results show that the error $\left\|y\left(x, P_{N}\right)-y_{0}\right\|$ goes down as the sample size $N$ increases and/or $\left\|x-x_{0}\right\|$ tends to zero, which confirms the stability of the problem as guaranteed by Theorem 5.1 . 


\subsection{Mathematical programs with SQVIP constraints}

Let us now consider a mathematical program with SQVIP constraint:

$$
\begin{array}{ll}
\min _{x, y} & f(x, y) \\
\text { s.t. } & 0 \in \mathbb{E}_{P}[F(x, y, \xi)]+\mathcal{N}_{\Gamma_{P}(x, y)}(y), \\
& (x, y) \in \mathcal{X} \times \mathcal{Y},
\end{array}
$$

where $f, F$ are continuous functions mapping from $\mathbb{R}^{n} \times \mathbb{R}^{m}$ and $\mathbb{R}^{n} \times \mathbb{R}^{m} \times \mathbb{R}^{k}$ to $\mathbb{R}$ and $\mathbb{R}^{m}$ respectively, $\xi$ is a random variable and $\Gamma_{P}(x, y)$ is defined as in (1.2). In the case when $\Gamma_{P}(x, y)$ is a constant convex set, (5.36) is known as a one stage stochastic mathematical program with equilibrium constraint which has been well investigated over the past decade, see for instance [4].

Our interest here is in the case when $\Gamma_{P}(x, y)$ does depend on either $P, x, y$ or at least one of them so that our discussion does not overlap with the existing research in the literature. Indeed, the novelty and challenge of model (5.36) lie in the SQVIP which may represent an equilibrium arising from a generalized stochastic game or the first order optimality conditions of a stochastic programming problem. Stochastic leader multiple followers problems, stochastic bilevel programming problems can all be put in this framework.

To maximize the coverage of our results, we consider $\Gamma_{P}(x, y)$ to be defined as in (1.2), that is,

$$
\Gamma_{P}(x, y):=\left\{z \in \mathcal{Y}: \mathbb{E}_{P}[G(x, y, z, \xi)] \in \mathcal{K}\right\}
$$

but leave $F(x, y, \xi)$ unspecified as to whether it arises from optimality of a single stochastic decision making problem or a stochastic game. In what follows, we present a quantitative stability result for (5.36) by exploiting the established stability results for SQVIP in the preceding sections. The result has a potential to provide a theoretical foundation for various numerical schemes for a range of stochastic optimization and equilibrium problems where either $G$ is not continuously differentiable or it is complicated to characterize $\mathcal{N}_{\Gamma_{P}(x, y)}(y)$ in terms of the derivatives of $G$ and normal cone of $\mathcal{K}$.

Theorem 5.2 Let $\vartheta_{P}$ and $\vartheta_{Q}$ denote the optimal value of MPSQVIP (5.36) and its perturbation respectively. Let $\mathcal{X} \subseteq \mathbb{R}^{n}$ and $\mathcal{Y} \subseteq \mathbb{R}^{m}$ be compact sets. Assume: (a) $f$ is a Lipschitz function in $(x, y)$ with a constant $\kappa>0$, (b) Assumption 3.1 holds at $x_{0} \in \mathcal{X}$ and Assumptions 3.2 holds for $F(x, y, \xi)$ and $G(x, y, z, \xi)$ respectively, (c) for $x_{0} \in \mathcal{X}, \mathbf{F}_{P}^{\text {nat }}\left(x_{0}, y\right)$ is a locally Lipschitz homeomorphism at every $y^{*} \in S_{P}\left(x_{0}\right)$. Then there exist positive constants $L, \delta$ and $\beta$ such that

$$
\left|\vartheta_{P}-\vartheta_{Q}\right| \leq L \mathscr{D}_{H}(Q, P)^{\beta}
$$

for any $Q \in U(P, \delta)$.

Proof. Let $\left(x_{P}^{*}, y_{P}^{*}\right)$ and $\left(x_{Q}^{*}, y_{Q}^{*}\right)$ denote the optimal solutions of (5.36) and its perturbation respectively and $\vartheta_{P}$ and $\vartheta_{Q}$ the corresponding optimal values. By Theorem 4.1 (iii), there exist positive constants $c_{1}, \beta, \delta_{1}$ and a neighborhood $\mathcal{V}$ of $y_{P}^{*}$ such that

$$
\left\|y-y_{P}^{*}\right\| \leq c_{1}\left(\mathscr{D}_{H}(Q, P)+\left\|x-x_{P}^{*}\right\|\right)^{\beta}
$$

for any $y \in S_{Q}(x) \cap \mathcal{V}$ with $Q \in U\left(P, \delta_{1}\right)$ and $x \in B_{n}\left(x_{P}^{*}, \delta_{1}\right) \cap \mathcal{X}$. In particular, for $z_{P}^{*} \in S_{Q}\left(x_{P}^{*}\right) \cap \mathcal{V}$,

$$
\left\|z_{P}^{*}-y_{P}^{*}\right\| \leq c_{1} \mathscr{D}_{H}(Q, P)^{\beta} \text {. }
$$


Consequently

$$
\begin{aligned}
\vartheta_{Q} & \leq f\left(x_{P}^{*}, z_{P}^{*}\right) \leq f\left(x_{P}^{*}, y_{P}^{*}\right)+\left\|f\left(x_{P}^{*}, z_{P}^{*}\right)-f\left(x_{P}^{*}, y_{P}^{*}\right)\right\| \\
& \leq \vartheta_{P}+\kappa\left\|z_{P}^{*}-y_{P}^{*}\right\| \leq \vartheta_{P}+\kappa c_{1} \mathscr{D}_{H}(Q, P)^{\beta},
\end{aligned}
$$

where $c_{1}$ is the Lipschitz modulus of $f$.

Likewise, for $z_{Q}^{*} \in S_{P}\left(x_{Q}^{*}\right)$, since $\mathbf{F}_{P}^{\text {nat }}\left(x_{Q}^{*}, y\right)$ is a locally Lipschitz homeomorphism at $z_{Q}^{*}$, by Theorem 4.1 (iii), there exist $c_{2}>0, \beta>0, \delta_{2}>0$ and a neighborhood $\mathcal{V}$ of $z_{Q}^{*}$ such that

$$
\left\|y-z_{Q}^{*}\right\| \leq c_{2}\left(\mathscr{D}_{H}(Q, P)+\left\|x-x_{Q}^{*}\right\|\right)^{\beta}
$$

for $y \in S_{Q}(x) \cap \mathcal{V}$ with $Q \in U\left(P, \delta_{2}\right)$ and $x \in B_{n}\left(x_{Q}^{*}, \delta_{2}\right) \cap \mathcal{X}$. In particular, for $y_{Q}^{*} \in S_{Q}\left(x_{Q}^{*}\right) \cap \mathcal{V}$,

$$
\left\|y_{Q}^{*}-z_{Q}^{*}\right\| \leq c_{2} \mathscr{D}_{H}(Q, P)^{\beta}
$$

Consequently we have

$$
\begin{aligned}
\vartheta_{P} & \leq f\left(x_{Q}^{*}, z_{Q}^{*}\right) \leq f\left(x_{Q}^{*}, y_{Q}^{*}\right)+\left\|f\left(x_{Q}^{*}, z_{Q}^{*}\right)-f\left(x_{Q}^{*}, y_{Q}^{*}\right)\right\| \\
& \leq \vartheta_{Q}+\kappa\left\|z_{Q}^{*}-y_{Q}^{*}\right\| \leq \vartheta_{Q}+\kappa c_{2} \mathscr{D}_{H}(Q, P)^{\beta} .
\end{aligned}
$$

Combining (5.38) and (5.40), we obtain the conclusion with $\delta=\min \left\{\delta_{1}, \delta_{2}\right\}$.

Acknowledgements. We would like to thank the editor-in-chief and two anonymous referees for valuable comments which help us significantly consolidate the paper.

\section{References}

[1] A. V. Arutyunov and A. F. Izmailov, Sensitivity analysis for cone-constrained optimization problems under the relaxed constraint qualifications, Mathematics of Operations Research, Vol. 30, pp. 333-353, 2005.

[2] R. J. Aumann, Integrals of set-valued functions, Journal of Mathematical Analysis and Applications, Vol. 12, pp. 1-12, 1965.

[3] J. P. Aubin and H. Frankowska, Set-Valued Analysis, Birkhäuser, 1990.

[4] S. Birbil, G. Gürkan and O. Listes, Solving stochastic mathematical programs with complementarity constraints using simulation, Mathematics of Operations Research, Vol. 31, pp. 739-760, 2006.

[5] J. F. Bonnans and A. Shapiro, Perturbation Analysis of Optimization Problems, Springer, New York, 2000.

[6] D. Chan and J. S. Pang, The generalized quasi-variational inequality problem, Mathematics of Operations Research, Vol. 7, pp. 211-222, 1982.

[7] X. Chen and M. Fukushima, Expected residual minimization method for stochastic linear complementarity problems, Mathematics of Operations Research, Vol. 30, pp. 1022-1038, 2005.

[8] X. Chen, R. B-J Wets and Y. Zhang, Stochastic variational inequalities: residual minimization smoothing/sample average approximations, SIAM Journal on Optimization, Vol. 22, pp. 649-673, 2012 . 
[9] C. Ding, D. F. Sun, L. W. Zhang, Characterization of the robust isolated calmness for a class of conic programming problems, http://arxiv.org/abs/1601.07418v1, 2016.

[10] J. Fiala, M. Kocvara and M. Stingl, PENLAB: A MATLAB solver for nonlinear semidefinite optimization, http://arxiv.org/abs/1311.5240, 2013.

[11] F. Facchinei, and J. S. Pang, Finite-Dimensional Variational Inequalities and Complementarity Problems I-II, Springer, New York, 2003.

[12] A. F. Izmailov, A. S. Kurennoy and M. V. Solodov, A note on upper Liptschitz stability, error bounds and critical multipliers for Liptschitz-continuous KKT systems, Mathematical Programming, Vol. 142, pp. 591-604, 2013.

[13] H. Jiang and H. Xu, Stochastic approximation approaches to the stochastic variational inequality problem, IEEE Transactions on Automatic Control, Vol. 53, pp. 1462-1475, 2008.

[14] A. J. King and R. T. Rockafellar, Sensitivity analysis for nonsmooth generalized equations, Mathematical Programming, Vol. 55, pp. 193-212, 1992.

[15] A. J. King and R. T. Rockafellar, Asymptotic theory for solutions in statistical estimation and stochastic programming, Mathematics of Operations Research, Vol. 18, pp. 148-162, 1993.

[16] D. Klatte, On the stability of local and global optimal solutions in parametric problems of nonlinear programming, Part I: Basic results, Humboldt-Universität Sektion Mathematik, Vol. 75, pp. 1-21, 1985.

[17] D. Klatte and B. Kummer, Nonsmooth Equations in Optimization: Regularity, Calculus, Methods and Applications, Kluwer, Dordrecht, 2002.

[18] Y. Liu, W. Römisch and H. Xu, Quantitative stability analysis of stochastic generalized equations, SIAM Journal on Optimization, Vol. 24, pp. 467-497, 2014.

[19] Y. Liu, H. Xu and G. H. Lin, Stability analysis of two stage stochastic mathematical programs with complementarity constraints via NLP-regularization, SIAM Journal on Optimization, Vol. 21, pp. 609-705, 2011.

[20] S. Lu and A. Budhiraja, Confidence regions for stochastic variational inequalities, Mathematics of Operations Research, Vol. 38, pp. 545-568, 2013.

[21] B. S. Mordukhovich and J. V. Outrata, Coderivative analysis of quasi-variational inequalities with applications to stability and optimization, SIAM Journal on Optimization, Vol. 18, pp. 389-412, 2007.

[22] J. S. Pang and M. Fukushima, Quasi-variational inequalities, generalized Nash equilibria, and multi-leader-follower games, Computational Management Science, Vol. 2, pp. 21-56, 2005.

[23] G. Ch. Pflug, Stochastic optimization and statistical inference. In: A. Ruszczynski and A. Shapiro (eds.), Stochastic Programming (Handbooks in Operations Research and Management Science, Vol. 10, pp. 427-480, Elsevier, Amsterdam, 2003.

[24] G. Ch. Pflug and A. Pichler, Approximations for probability distributions and stochastic optimization problems. In: M. Bertocchi, G. Consigli and M. A. Dempster (eds.), Stochastic Optimization Methods in Finance and Energy, Vol. 163, pp. 343-387, Springer, New York, 2011. 
[25] U. Ravat and U. V. Shanbhag, On the characterization of solution sets of smooth and nonsmooth convex stochastic Nash games, SIAM Journal on Optimization, Vol. 21, pp. 1168-1199, 2011.

[26] R. T. Rockafellar and R. J. B. Wets, Variational Analysis, Berlin Heidelberg, 1998.

[27] S. M. Robinson, An application of error bounds for convex programming in linear space, SIAM Journal of Control and Optimization, Vol. 13, pp. 271-273, 1975.

[28] S. M. Robinson, Stability theory for systems of inequalities, Part II: Differentiable nonlinear systems, SIAM Journal on Numerical Analysis, Vol. 13, pp. 497-513, 1976.

[29] S. M. Robinson, Generalized equations and their solutions, Part I: Basic theory, Mathematical Programming Study, Vol. 10, pp. 128-141, 1979.

[30] S. M. Robinson, Strongly regular generalized equations, Mathematics of Operations Research, Vol. 5, pp. 43-62, 1980.

[31] S. M. Robinson, Generalized equations and their solutions, Part II: Applications to nonlinear programming, Mathematical Programming Study, Vol. 19, pp. 200-221, 1982.

[32] R. T. Rockafellar and R. J.B. Wets, Stochastic variational inequalities: single-stage to multistage, Mathematical Programming, 2016.

[33] W. Römisch, Stability of stochastic programming problems. In: A. Ruszczynski, A. Shapiro (eds.), Stochastic Programming. Handbooks in Operations Research and Management Science, Vol. 10, pp. 483-554. Elsevier, Amsterdam, 2003.

[34] A. Rusczynski, A. Shapiro, Stochastic Programming, Handbooks in OR \& MS, Elsevier, Amsterdam, 2003.

[35] A. Shapiro, Sensitivity analysis of parameterized variational inequalities, Mathematics of Operations Research, Vol. 30, pp. 109-126, 2005.

[36] A. Shapiro, Sensitivity analysis of generalized equations, Journal of Mathematical Sciences, Vol.115, pp. 2554-2565, 2003.

[37] A. Shapiro, D. Dentcheva and A. Ruszczynski, Lectures on Stochastic Programming: Modeling and Theory, SIAM, Philadelphia, 2009.

[38] J. Wolfowitz, Generalization of the theorem of glivenko-cantelli, Annals of Mathematical Statistics, Vol. 25, pp. 131-138, 1954.

[39] H. Xu, Sample average approximation methods for a class of stochastic variational inequality problems, Asian Pacific Journal of Operations Research, Vol. 27, pp. 103-119, 2010.

[40] H. Xu and J. J. Ye, Approximating stationary points of stochastic mathematical programs with variational inequality constraints via sample averaging, Set-Valued and Variational Analysis, Vol. 19, pp. 283-309, 2011.

[41] F. Yousefian, A. Nedić and U. V. Shanbhag, A regularized smoothing stochastic approximation (RSSA) algorithm for stochastic variational inequality problems, Proceeding WSC '13 Proceedings of the 2013 Winter Simulation Conference: Simulation: Making Decisions in a Complex World, 933-944, 2013. 
[42] F. Yousefian, A Nedich and U Shanbhag, Self-Tuned Stochastic Approximation Schemes for Non-Lipschitzian Stochastic Multi-User Optimization and Nash Games, IEEE Transactions on Automatic Control, Vol. 61, pp. 1753-1766, 2016.

\section{Appendix}

Proof of Lemma 2.2. Part (i). Observe that (2.4) implies that $\Psi(\bar{u}) \neq \emptyset$, and under uniform continuity w.r.t. $u$ further that $\Psi(u) \neq \emptyset$ when $u$ is sufficiently close to $\bar{u}$.

It is well known (see [3, Definition 1.4.6]) that

$$
\liminf _{u \rightarrow \bar{u}} \Psi(u) \subseteq \operatorname{cl} \Psi(\bar{u}) \subseteq \limsup _{u \rightarrow \bar{u}} \Psi(u)
$$

Since $\Psi(\bar{u})$ is closed, to show continuity of $\Psi(\cdot)$ at $\bar{u}$, it suffices to show that the set-valued mapping is both upper and lower semicontinuous at $\bar{u}$, that is,

$$
\limsup _{u \rightarrow \bar{u}} \Psi(u) \subseteq \Psi(\bar{u}) \subseteq \liminf _{u \rightarrow \bar{u}} \Psi(u) .
$$

The upper semicontinuity can be easily verified under the continuity of $\psi$ and closeness of $\mathcal{K}$. Hence it suffices to show the lower semicontinuity.

Observe that condition (2.4) and continuity of $\psi(\cdot, \bar{u})$ imply that int $\Psi(\bar{u}) \neq \emptyset$. Moreover, since $\psi(z, u)$ is uniformly continuous w.r.t. $u$ under condition (b), it is easy to show that for any $z \in$ int $\Psi(\bar{u})$, we can set $u$ sufficiently close to $\bar{u}$ such that $z \in \Psi(u)$. This allows us to claim that

$$
\text { int } \Psi(\bar{u}) \subseteq \liminf _{u \rightarrow \bar{u}} \Psi(u) .
$$

Moreover, since $\Psi(\bar{u})$ is convex and closed and by [26, Proposition 4.4], $\liminf _{u \rightarrow \bar{u}} \Psi(u)$ is closed, we have

$$
\Psi(\bar{u})=\operatorname{cl}(\operatorname{int} \Psi(\bar{u})) \subseteq \operatorname{cl}\left(\liminf _{u \rightarrow \bar{u}} \Psi(u)\right)=\liminf _{u \rightarrow \bar{u}} \Psi(u),
$$

which yields the second inclusion of (5.41).

Part (ii). Since $\Psi(\bar{u})$ is compact and $\Psi(\cdot)$ is closed at $\bar{u}$, the continuity of $\Psi(\cdot)$ at $\bar{u}$ means that for any number $\delta>0$, there exists $\epsilon>0$ such that when $\|u-\bar{u}\|_{U} \leq \epsilon$,

$$
\mathbb{H}(\Psi(u), \Psi(\bar{u})) \leq \delta
$$

or equivalently

$$
\Psi(u) \subseteq \Psi(\bar{u})+\delta \mathcal{B}_{m} \text { and } \Psi(\bar{u}) \subseteq \Psi(u)+\delta \mathcal{B}_{m} .
$$

In what follows, we use Lemma 2.1 (Robinson-Ursescu's theorem) to derive an error bound for setvalued mapping $\Psi(\cdot)$ at $\bar{u}$ under condition (d) and Slater constraint qualification (2.4).

Let

$$
\mathcal{F}_{u}(z):=\left\{\begin{array}{cl}
\psi(z, u)-\mathcal{K}, & \text { for } z \in \mathcal{Z} \\
\emptyset, & \text { for } z \notin \mathcal{Z}
\end{array}\right.
$$


Condition (a) ensures that $\mathcal{F}_{u}(z)$ is closed and convex set-valued over its domain $\mathcal{Z}$. Moreover,

$$
\Psi(u)=\mathcal{F}_{u}^{-1}(0)
$$

and $z \in \Psi(u)$ if and only if $(z, 0) \in \operatorname{gph} \mathcal{F}_{u}$. Furthermore, it follows from (2.4)

$$
0 \in \operatorname{int}\left(\text { range } \mathcal{F}_{\bar{u}}\right) \text {. }
$$

Let $z \in \Psi(\bar{u})$ be fixed, that is, $(z, 0) \in \operatorname{gph} \mathcal{F}_{\bar{u}}$. By Lemma 2.1, there exist positive numbers $\delta_{z}, \eta_{z}$ and $c_{z}$ (depending on $z$ ) such that

$$
d\left(z^{\prime}, \mathcal{F}_{\bar{u}}^{-1}(q)\right) \leq c_{z} d\left(q, \mathcal{F}_{\bar{u}}\left(z^{\prime}\right)\right)
$$

for each $z^{\prime} \in B_{m}\left(z, \delta_{z}\right) \cap \mathcal{Z}$ and $q \in B_{W}\left(0, \eta_{z}\right)$. In particular,

$$
d\left(z^{\prime}, \Psi(\bar{u})\right)=d\left(z^{\prime}, \mathcal{F}_{\bar{u}}^{-1}(0)\right) \leq c_{z} d\left(\psi\left(z^{\prime}, \bar{u}\right), \mathcal{K}\right) .
$$

Under condition (d), we have

$$
d\left(\psi\left(z^{\prime}, \bar{u}\right), \mathcal{K}\right) \leq\left\|\psi\left(z^{\prime}, \bar{u}\right)-\psi\left(z^{\prime}, u\right)\right\|_{Z}+d\left(\psi\left(z^{\prime}, u\right), \mathcal{K}\right) \leq \sigma\|u-\bar{u}\|_{U}^{\nu}
$$

for $z^{\prime} \in \Psi(u)$.

On the other hand, since $z$ is arbitrarily chosen from $\Psi(\bar{u})$, the set $\Psi(\bar{u})$ may be covered by the union of a collection of $\delta$-balls, i.e.,

$$
\Psi(\bar{u}) \subseteq \bigcup_{z \in \Psi(\bar{u})} \operatorname{int} B_{m}\left(z, \delta_{z}\right)
$$

Since $\Psi(\bar{u})$ is a compact set, by the finite covering theorem, there exist a finite number of points $z_{1}, z_{2}, \cdots, z_{k} \in \Psi(\bar{u})$ and positive constants $\delta_{z_{i}}, c_{z_{i}}, i=1, \cdots, k$ such that

$$
\Psi(\bar{u}) \subseteq \bigcup_{i=1}^{k} \operatorname{int} B_{m}\left(z_{i}, \delta_{z_{i}}\right)
$$

and

$$
d\left(z^{\prime}, \Psi(\bar{u})\right) \leq c_{z_{i}} d\left(\psi\left(z^{\prime}, \bar{u}\right), \mathcal{K}\right)
$$

for any $z^{\prime} \in B_{m}\left(z_{i}, \delta_{z_{i}}\right) \cap \mathcal{Z}$. Since $\Psi(\bar{u})$ is a compact set and $\bigcup_{i=1}^{k}$ int $B_{m}\left(z_{i}, \delta_{z_{i}}\right)$ is an open set, there exists a positive constant $\delta$ such that

$$
\Psi(\bar{u})+\delta \mathcal{B}_{m} \subseteq \bigcup_{i=1}^{k} \operatorname{int} B_{m}\left(z_{i}, \delta_{z_{i}}\right) .
$$

Let $C:=\max \left\{c_{z_{1}}, \cdots, c_{z_{k}}\right\}$. Combining (5.51) and (5.50), we obtain

$$
d\left(z^{\prime}, \Psi(\bar{u})\right) \leq C d\left(\psi\left(z^{\prime}, \bar{u}\right), \mathcal{K}\right)
$$

for all $z^{\prime} \in\left(\Psi(\bar{u})+\delta \mathcal{B}_{m}\right) \cap \mathcal{Z}$. Through (5.43) and (5.48), we arrive at

$$
\mathbb{D}(\Psi(u), \Psi(\bar{u})) \leq C d(\psi(z, \bar{u}), \mathcal{K}) \leq C \sigma\|u-\bar{u}\|_{U}^{\nu}
$$


for all $z^{\prime} \in \Psi(u)$.

To complete the proof of $(2.5)$, it suffices to show

$$
\mathbb{D}(\Psi(\bar{u}), \Psi(u)) \leq C \sigma\|u-\bar{u}\|_{U}^{\nu} .
$$

For any $z \in \mathcal{Z}$, let $q=\psi(z, \bar{u})-\psi(z, u)$. Under condition (d), $\|q\|_{W} \leq \sigma\|u-\bar{u}\|_{U}^{\nu}$. Moreover, it is easy to see that $\Psi(u)=\mathcal{F}_{\bar{u}}^{-1}(q)$. Let $z \in \Psi(\bar{u}), \delta_{z}$ be defined as in (5.46) and $\epsilon$ be defined at the beginning of the proof of this part with $\sigma \epsilon^{\nu} \leq \delta_{z}$. Under condition (d), we have $\|q\| \leq \delta_{z}$. It follows from (5.46) (by setting $z^{\prime}=z$ ) that

$$
d(z, \Psi(u))=d\left(z, \mathcal{F}_{\bar{u}}^{-1}(q)\right) \leq c_{z} d\left(q, \mathcal{F}_{\bar{u}}(z)\right) .
$$

Since $z \in \Psi(\bar{u})$, then $0 \in \mathcal{F}_{\bar{u}}(z)$ and hence

$$
d\left(q, \mathcal{F}_{\bar{u}}(z)\right) \leq\|q\|_{W} \leq \sigma\|u-\bar{u}\|_{U}^{\nu} .
$$

Combining the two inequalities above, we obtain

$$
d(z, \Psi(u)) \leq c_{z} \sigma\|u-\bar{u}\|_{U}^{\nu}
$$

for any $z \in \Psi(\bar{u})$ and $\|u-\bar{u}\|_{U} \leq \epsilon$. Utilizing the second inclusion in (5.43), we can apply the finite covering theorem to set $\Psi(\bar{u})$ (similar to previous discussion) and find a positive constant $C$ such that (5.53) holds. The proof is complete.

In the case when $\psi$ is continuously differentiable, Lemma 2.2 coincides with [28, Corollary 1]. Thus we may regard our result as an extension of the Robinson's result.

Proof of Lemma 2.3. Let $\hat{\phi}(z, u):=\phi(z, u)+\delta_{\Psi(u)}(z)$, where $\delta_{C}(\cdot)$ denotes the indicator function of a set $C \subseteq \mathbb{R}^{m}$ with $\delta_{C}(x)=0$ for $x \in C$ and $\delta_{C}(x)=+\infty$ for $x \notin C$. Problem (2.3) can be equivalently written as

$$
\min _{z \in \mathbb{R}^{m}} \hat{\phi}(z, u) .
$$

The objective function $\hat{\phi}$ has the following properties:

(i) Since $\phi$ and $\psi$ are continuous, $\mathcal{K}$ and $\mathcal{Z}$ are closed and convex, we have graph $\Psi$ is closed, which means that epi $\delta_{\Psi(\cdot)}(\cdot)=\{(u, z, \alpha): z \in \Psi(u), \alpha \geq 0\}=\operatorname{graph} \Psi \times \mathbb{R}_{+}$is closed. Hence $\hat{\phi}$ is proper lower semicontinuous;

(ii) Since $\mathcal{Z}$ is compact, $\hat{\phi}$ is level-bounded in $z$ locally uniformly in $u$;

(iii) Under the Slater condition (2.4), one has from Lemma 2.2 that

$$
\lim _{u \rightarrow \bar{u}} \Psi(u)=\Psi(\bar{u})
$$

which means that

$$
\limsup _{u \rightarrow \bar{u}} \operatorname{epi}\left[\delta_{\Psi(u)}(\cdot)\right]=\operatorname{epi}\left[\delta_{\Psi(\bar{u})}(\cdot)\right]
$$

where

$$
\operatorname{epi}\left[\delta_{\Psi(u)}(\cdot)\right]=\{(z, \alpha): z \in \Psi(u), \alpha \geq 0\}=\Psi(u) \times \mathbb{R}_{+} .
$$

Then we obtain from Definition 2.1 that

$$
e-\lim _{u \rightarrow \bar{u}} \delta_{\Psi(u)}(\cdot)=\delta_{\Psi(\bar{u})}(\cdot),
$$


which, by Proposition 2.1, means that for any sequence $u^{\nu} \rightarrow \bar{u}$ and point $\bar{z} \in \mathbb{R}^{m}$,

$$
\liminf _{\nu \rightarrow \infty} \delta_{\Psi\left(u^{\nu}\right)}\left(z^{\nu}\right) \geq \delta_{\Psi(\bar{u})}(\bar{z})
$$

for every sequence $z^{\nu} \rightarrow \bar{z}$ and there exists some $z^{\nu} \rightarrow \bar{z}$ such that

$$
\limsup _{\nu \rightarrow \infty} \delta_{\Psi\left(u^{\nu}\right)}\left(z^{\nu}\right) \leq \delta_{\Psi(\bar{u})}(\bar{z})
$$

We know from the continuity of $\phi$ that

$$
\lim _{\nu \rightarrow \infty} \phi\left(z^{\nu}, u^{\nu}\right)=\phi(\bar{z}, \bar{u}) \text { as } \nu \rightarrow \infty
$$

which leads to

$$
\liminf _{\nu \rightarrow \infty}\left[\phi\left(z^{\nu}, u^{\nu}\right)+\delta_{\Psi\left(u^{\nu}\right)}\left(z^{\nu}\right)\right] \geq \phi(\bar{z}, \bar{u})+\delta_{\Psi(\bar{u})}(\bar{z})
$$

and there exists $z^{\nu} \rightarrow \bar{z}$ such that

$$
\limsup _{\nu \rightarrow \infty}\left[\phi\left(z^{\nu}, u^{\nu}\right)+\delta_{\Psi\left(u^{\nu}\right)}\left(z^{\nu}\right)\right] \leq \phi(\bar{z}, \bar{u})+\delta_{\Psi(\bar{u})}(\bar{z}) .
$$

Then also by Proposition 2.1, we have that $u \mapsto \hat{\phi}(\cdot, u)$ is epi-continuous at $\bar{u}$.

Hence all conditions in [26, Theorem 7.41] are satisfied, and from this theorem we obtain that $\vartheta(\cdot)$ is continuous at $\bar{u}$ and $\mathcal{Z}(\cdot)$ is outer semicontinuous at $\bar{u}$. Noting that $\mathcal{Z}(u) \subset \mathcal{Z}$ and it actually is locally bounded around $\bar{u}$, we have from $[26$, Theorem 5.19] that $\mathcal{Z}(\cdot)$ is upper semicontinuous at $\bar{u} \cdot \square$

Proof of Theorem 2.1. Part (i). First we show that

$$
\mathbb{D}(\mathcal{Z}(u), \mathcal{Z}(\bar{u})) \leq \max _{z \in \mathcal{Z}(u)}\left\|z-\Pi_{\Psi(\bar{u})}(z)\right\|+R(u),
$$

where $\Pi_{\Psi(\bar{u})}(z)$ denotes orthogonal projection of $z$ on $\Psi(\bar{u})$,

$$
R(u):=\left(\frac{1}{\alpha}\left[2 \varrho\|u-\bar{u}\|_{U}^{\gamma}+L\left(\max _{z \in \mathcal{Z}(u)}\left\|z-\Pi_{\Psi(\bar{u})}(z)\right\|+\min _{z \in \mathcal{Z}(\bar{u})}\left\|z-\Pi_{\Psi(u)}(z)\right\|\right)\right]\right)^{\frac{1}{2}} .
$$

Let $z(u) \in \mathcal{Z}(u)$ and $z(\bar{u}) \in \mathcal{Z}(\bar{u})$. Under conditions (b) and (c)

$$
\begin{aligned}
\vartheta(u)-\phi\left(\Pi_{\Psi(\bar{u})}(z(u)), \bar{u}\right) & =\phi(z(u), u)-\phi\left(\Pi_{\Psi(\bar{u})}(z(u)), \bar{u}\right) \\
& \geq-L\left\|z(u)-\Pi_{\Psi(\bar{u})}(z(u))\right\|-\varrho\|u-\bar{u}\|_{U}^{\gamma}
\end{aligned}
$$

for $u \in B\left(\bar{u}, \bar{\delta}_{u}\right)$. On the other hand, since $\Pi_{\Psi(u)}(z(\bar{u})) \in \Psi(u)$ and $z(u)$ is an optimal solution to $\mathcal{P}_{u}$, we have

$$
\vartheta(u) \leq \phi\left(\Pi_{\Psi(u)}(z(\bar{u})), u\right) .
$$

Using this inequality, (2.8) and the growth condition (c), we have

$$
\begin{aligned}
\vartheta(u)-\phi\left(\Pi_{\Psi(\bar{u})}(z(u)), \bar{u}\right)= & \phi(z(u), u)-\phi(z(\bar{u}), u)+(\phi(z(\bar{u}), u)-\phi(z(\bar{u}), \bar{u})) \\
& -\left(\phi\left(\Pi_{\Psi(\bar{u})}(z(u)), \bar{u}\right)-\phi(z(\bar{u}), \bar{u})\right) \\
\leq & \phi\left(\Pi_{\Psi(u)}(z(\bar{u})), u\right)-\phi(z(\bar{u}), u)+\varrho\|u-\bar{u}\|_{U}^{\gamma} \\
& -\alpha d\left(\Pi_{\Psi(\bar{u})}(z(u)), \mathcal{Z}(\bar{u})\right)^{2} \\
\leq & L\left\|\Pi_{\Psi(u)}(z(\bar{u}))-z(\bar{u})\right\|+\varrho\|u-\bar{u}\|_{U}^{\gamma} \\
& -\alpha d\left(\Pi_{\Psi(\bar{u})}(z(u)), \mathcal{Z}(\bar{u})\right)^{2}
\end{aligned}
$$


for $u \in B\left(\bar{u}, \bar{\delta}_{u}\right)$. Combining (5.57) and (5.58), we obtain

$$
\begin{aligned}
& d\left(\Pi_{\Psi(\bar{u})}(z(u)), \mathcal{Z}(\bar{u})\right) \\
& \leq\left(\left[L\left\|z(u)-\Pi_{\Psi(\bar{u})}(z(u))\right\|+2 \varrho\|u-\bar{u}\|_{U}^{\gamma}+L\left\|z(\bar{u})-\Pi_{\Psi(u)}(z(\bar{u}))\right\|\right] / \alpha\right)^{\frac{1}{2}},
\end{aligned}
$$

which yields (5.55) through the triangle inequality below

$$
d(z(u), \mathcal{Z}(\bar{u})) \leq\left\|z(u)-\Pi_{\Psi(\bar{u})}(z(u))\right\|+d\left(\Pi_{\Psi(\bar{u})}(z(u)), \mathcal{Z}(\bar{u})\right)
$$

because $z(u)$ and $z(\bar{u})$ are arbitrarily taken from the set of optimal solutions. Since $\mathcal{Z}(u) \subseteq \Psi(u)$, it follows by Lemma 2.2 that there exists a constant $\epsilon>0$ such that

$$
\max \left\{\max _{z \in \mathcal{Z}(u)}\left\|z-\Pi_{\Psi(\bar{u})}(z)\right\|, \min _{z \in \mathcal{Z}(\bar{u})}\left\|z-\Pi_{\Psi(u)}(z)\right\|\right\}=O\left(\|u-\bar{u}\|_{U}^{\nu}\right)
$$

when $\|u-\bar{u}\|_{U} \leq \epsilon$. Combining (5.55) and (5.61), we obtain (2.9).

Part (ii). We only need to show that

$$
\mathbb{D}(\mathcal{Z}(\bar{u}), \mathcal{Z}(u)) \leq c^{\prime}\|u-\bar{u}\|_{U}^{\beta} .
$$

From the proof of Part (i), we can see that we can swap $u$ with $\bar{u}$ except that the first inequality in formulae (5.58) requires second order growth condition of $\phi(\cdot, u)$ over the optimal solution set $\mathcal{Z}(u)$. 